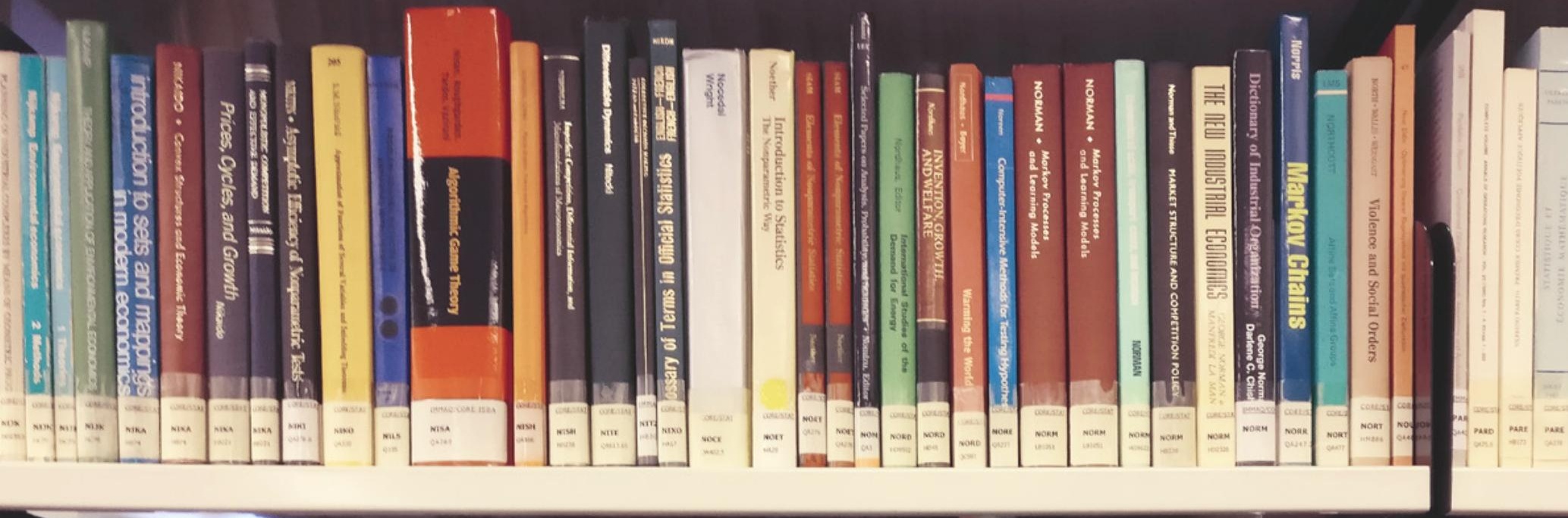

$2017 / 16$

DP

Mathieu Lefèbvre, Pierre Pestieau and Gregory Ponthiere

Premature mortality and poverty measurement in an

OLG economy

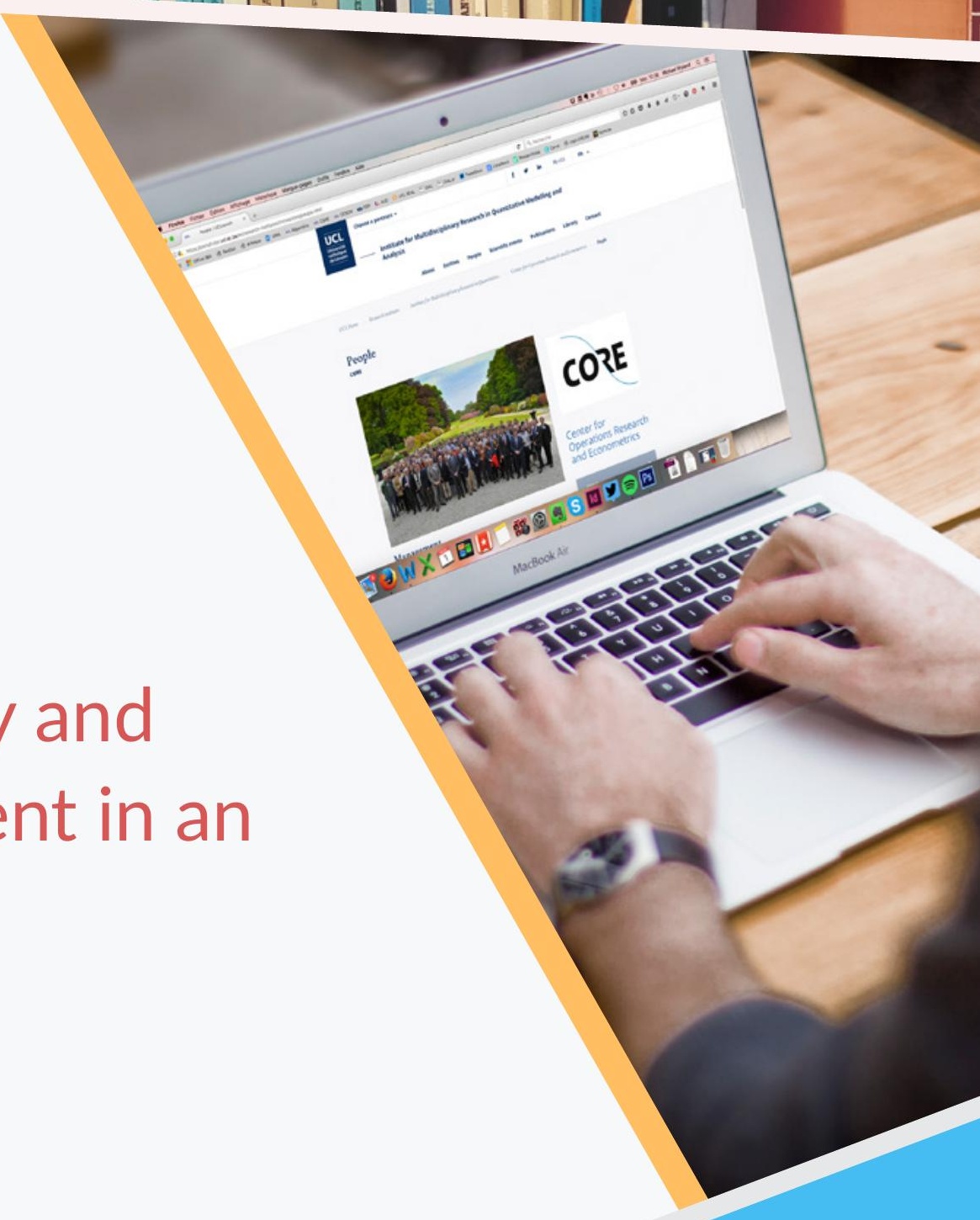




\section{CORE}

Voie du Roman Pays 34, L1.03.01

Tel (32 10) 474304

Fax (32 10) 474301

Email: immaq-library@uclouvain.be

https://uclouvain.be/en/research-institutes/

immaq/core/discussion-papers.html 


\title{
Premature Mortality and Poverty Measurement in an OLG Economy
}

\author{
Mathieu Lefèbvre* ${ }^{*}$ Pierre Pestieau ${ }^{\dagger}$ Gregory Ponthiere ${ }^{\ddagger}$
}

April 24, 2017

\begin{abstract}
Following Kanbur and Mukherjee (2007), a solution to the "missing poor" problem (i.e. selection bias in poverty measures due to incomedifferentiated mortality) consists in computing hypothetical poverty rates while assigning a fictitious income to the prematurely dead. However, in a dynamic general equilibrium economy, doing "as if" the prematurely dead were still alive is likely to affect wages, output and capital accumulation, with an uncertain effect on poverty. We develop a 3-period OLG model with income-differentiated mortality, and compare actual poverty rates with hypothetical poverty rates that would have prevailed if everyone faced the survival conditions of the top income class. Including the prematurely dead has an ambiguous impact on poverty, since it affects income distribution through capital dilution, composition effects and horizon effects. Our results are illustrated by quantifying the impact of incomedifferentiated mortality on poverty measures for France (1820-2010).

Keywords: income-differentiated mortality, poverty measures, missing poor, OLG models, capital accumulation.

JEL classification code: E13, E21, I32.
\end{abstract}

\footnotetext{
*University of Strasbourg, BETA.

${ }^{\dagger}$ University of Liege, CORE and Paris School of Economics.

$\ddagger$ University Paris East (ERUDITE), Paris School of Economics and Institut universitaire de France. [corresponding author] Address: Ecole Normale Superieure, 48 bd Jourdan, 75014 Paris, France. E-mail: gregory.ponthiere@ens.fr
} 


\section{Introduction}

In his Essay on the Principle of Population, Thomas Malthus (1798) emphasized that poor households face worse survival conditions than non-poor households. Malthus coined that phenomenon the "positive population check", through which the population size adjusts to available resources. Whereas one can disagree with the Malthusian doctrine of population, it remains true that, two centuries after Malthus - and despite the rise of the Welfare State -, there is still a negative correlation between income and mortality. ${ }^{1}$

The positive relation between income and life expectancy raises deep difficulties for poverty measurement. As emphasized by Kanbur and Mukherjee (2007), standard poverty measures are, under income-differentiated mortality, biased downwards. Poor persons tend to die earlier than non-poor ones, so that a higher number of poor persons disappear from poverty statistics, leading to a selection bias. ${ }^{2}$ Following Sen's (1998) emphasis on "missing women", we can coin this the "missing poor" problem. Missing poor can bias poverty comparisons across countries and time periods. To illustrate this, suppose that the income/mortality gradient is stronger in economy $A$ than in economy $B$. Given that there are more missing poor in $A$ than in $B$, poverty measures may show a lower prevalence of poverty in $A$ than in $B$, but this lower measured poverty in "nominal terms" may not coincide with a lower actual poverty. ${ }^{3}$

Kanbur and Mukherjee (2007) proposed to solve the missing poor problem by assigning a fictitious income to the prematurely dead poor, in such a way as to reintroduce the missing poor in poverty statistics. Having assigned a fictitious income to the prematurely dead poor, and having adjusted the income distribution accordingly, one can then compute hypothetical poverty rates based on the new income distribution. As studied in Lefebvre et al (2013), that method admits several variants, depending on whether a fictitious income is assigned only to the prematurely dead poor, or, on the contrary, to any prematurely dead person. Another important issue concerns the level of fictitious incomes. One can assign, as a proxy, the income enjoyed by the prematurely dead when he used to be alive, or another, lower income level, taking into account the fact

\footnotetext{
${ }^{1}$ On this correlation, see Duleep (1986), Backlund et al (1999), Deaton and Paxson (1998), Deaton (2003), Salm (2007) and Belloni et al (2013). Note that the direction of causality has been much studied in the recent years, some studies finding no causal link from income to mortality (see Snyder and Evans, 2005; Evans and Moore, 2011; Ahammer et al, 2015).

2 The selection bias arises because of the income/mortality correlation, independently from any causality issue. To see this, suppose that an exogenous bus strike arises on the day of a classroom test, which prevents some students from attending the test. The average mark at the test (computed on students attending) suffers from a selection bias as soon as there is a correlation between potential marks and being victim of the bus strike, implying that the average mark would have been different provided no bus strike took place. This is true even if there is no causal link between being subject to a bus strike and potential marks.

${ }^{3}$ That case occurs in reality. Lefebvre et al (2017) show that, although poverty beyond age 60 is higher in Portugal than in Estonia, once we correct for the missing poor problem (by computing the poverty rates that would have prevailed if all income groups had faced the survival conditions of the richest), old-age poverty is higher in Estonia than in Portugal.
} 
that a premature death is a major source of deprivation. ${ }^{4}$

The method developed by Kanbur and Mukherjee supposes a kind of ceteris paribus postulate: it is assumed that adding the missing poor does not affect the incomes of other persons. This is a partial equilibrium approach, where the addition of the prematurely dead is supposed to leave the rest of the economy unchanged. However, in a dynamic general equilibrium economy, doing "as if" the prematurely dead were still alive is likely to affect wages, output and capital accumulation, with an uncertain effect on poverty. An important question is to know to what extent those general equilibrium effects reduce the downward bias due to income-differentiated mortality. Once we take general equilibrium effects into account, is it still true that adding the missing poor increases poverty? Or could it be the case that adding the missing poor reduces poverty?

This paper proposes to examine how the missing poor phenomenon affects the measurement of poverty in a dynamic general equilibrium economy. Our goal is twofold. First, we propose to identify the general equilibrium effects induced by income-differentiated mortality. Those general equilibrium effects can be identified by comparing the actual economy with what would arise in the hypothetical case where all income classes faced the same mortality risks. Second, we propose to identify conditions under which income-differentiated mortality contributes, in our general equilibrium setting, to increase or decrease measured poverty. For those purposes, we develop a 3-period dynamic overlapping generations (OLG) model with income-differentiated mortality, and examine to what extent abstracting from income-differentiated mortality would have led, within that dynamic general equilibrium model, to a different dynamics of production and capital accumulation, and, in fine, to different prevalences of poverty.

To identify the mechanisms at work, we first characterize the stationary equilibrium of our OLG economy including agents differing on labor productivity and survival conditions, and consider the impact of changing survival conditions on the stationary equilibrium. Then, in a second stage, we compare actual poverty measures with hypothetical poverty measures (assuming that all income groups face the same survival conditions as the top income group), in order to identify conditions under which income-differentiated mortality leads to a rise in measured poverty. Finally, in a third stage, we calibrate our model so as to fit the patterns of income and poverty in France (1820-2010), and we quantify the impact of the missing poor phenomenon on poverty measurement.

Anticipating our results, we first show that the inclusion of prematurely dead persons has several effects on capital accumulation and production, and, hence, on the distribution of income and the measured poverty. First of all, adding prematurely dead persons (by supposing that all income groups face the survival conditions of the richest) contributes to dilute capital, which raises poverty. Secondly, adding prematurely dead persons modifies the composition of the population in terms of savings behavior, by adding individuals who save less, which also raises poverty by lowering capital accumulation. However, there

\footnotetext{
${ }^{4}$ Lefebvre et al (2013) propose to take as a fictitious income the income equivalent to death, i.e. the income that would make a person indifferent between, on the one hand, remaining alive with that income level, and, on the other hand, dying.
} 
is a third effect, which plays in the opposite direction. Assuming that everyone faces the survival conditions of the top income group raises the expected lifespan of many individuals, which, in turn, favors savings and capital accumulation. This horizon effect contributes to reduce poverty. As a consequence, whether correcting for income-differentiated mortality leads to a higher or a lower prevalence of poverty depends on whether the capital dilution effect and savers composition effects dominate or not the horizon effect.

We show that, in theory, when the capital dilution effect dominates the horizon effect, the addition of prematurely dead persons increases measures of absolute poverty, implying that income-differentiated mortality tends to bias poverty measures downwards. ${ }^{5}$ When the horizon effect dominates other effects (so that adding missing persons raises the capital to labor ratio), it is not necessarily the case that adding missing persons reduces the extent of poverty. Having a strong horizon effect is thus a necessary, but not sufficient condition to imply that adding prematurely dead persons reduces measured poverty.

Our numerical application to France (1820-2010) shows that the capital dilution effect and the population composition effect dominated the horizon effect, implying that income-differentiated mortality contributed to lower measured poverty. Our numerical simulations also show that the extent to which incomedifferentiated mortality affects poverty measurement varies across periods, reflecting the varying strength of the income/mortality gradient. The size of the gap between hypothetical and actual poverty measures depends also on whether poverty is absolute or relative, and on the age group under study.

In sum, this paper shows that, once we consider a dynamic general equilibrium setting, correcting for the missing poor problem becomes more complex, and can have various effects on the measurement of poverty. When we take all those effects into account, it still appears that counting the missing poor leads to a rise in measured poverty, whose size depends on the extent of incomedifferentiated mortality. Put it differently, this paper shows that, if there had been no income-differentiated mortality in the past, poverty measures would have taken higher values, especially in the early 19th century. Thus the missing poor problem is robust to considering a dynamic general equilibrium framework.

This paper complements two branches of the literature, and proposes to build a kind of bridge between these. First, our study is related to previous works on poverty measurement under premature mortality, such as Kanbur and Mukherjee (2007) and Lefebvre et al $(2013,2017)$. Whereas those papers were based on a partial equilibrium approach, our analysis reframes the problem of missing poor in a dynamic general equilibrium framework. Our paper is also related to the literature on the link between mortality changes and longrun development, such as de la Croix and Licandro (1999), Boucekkine et al (2001), Blackburn and Cipriani (2002), Chakraborty (2004) and de la Croix and Licandro (2013). Our paper complements that literature by examining poverty measurement in an OLG economy where individuals differ in incomes, and where life expectancy is positively related to income.

\footnotetext{
${ }^{5}$ Note that we cannot make the same claim for measures of relative poverty.
} 
The paper is organized as follows. Section 2 presents the model. The longrun dynamics is examined in Section 3. Section 4 studies the impact of incomedifferentiated mortality on measures of absolute poverty at the stationary equilibrium. Section 5 examines the impact of income-differentiated mortality on measures of relative poverty. The historical example of France (1820-2010) is considered in Section 6. Section 7 concludes.

\section{The model}

We consider a 3-period OLG model with capital accumulation and risky lifetime. Period 1 is childhood, during which individuals do not make any decision. Period 2 is young adulthood, during which individuals work, consume, save and have $g>0$ children. Period 3 is the old age, during which individuals are retired.

Lifetime is risky: only a fraction $\phi$ of the newborn will survive to young adulthood, and only a fraction $\pi$ of young adults will reach the old age. Whereas the general structure of the model is in line with Chakraborty (2004) (except that we allow for premature death before becoming an adult), a major difference lies in the fact that we assume here that the population is not homogeneous, but, rather, differs in terms of innate human capital. ${ }^{6}$

\subsection{Heterogeneity}

There are three types of agents $i \in\{1,2,3\}$, who differ on their innate human capital $\lambda_{i} \cdot{ }^{7}$ The parameter $\lambda_{i}>0$ is positively related to both the labor productivity of the individual in case of survival to young adulthood and to the survival conditions faced by that individual during his life. The parameter $\lambda_{i}$ can thus be interpreted as human capital broadly defined, which includes health capital. The three types of agents are denoted in an increasing order in terms of innate human capital, that is:

$$
\lambda_{1}<\lambda_{2}<\lambda_{3}
$$

The distribution of the population into types within a given cohort at birth, that is, the relative number of the different types of individuals in each cohort of newborns, is supposed to be constant over time. The parameter $n_{i}$ denotes the proportion of potential individuals of type $i=1,2,3$. One can thus interpret the parameter $\lambda_{i}$ as the outcome of a lottery run by Nature concerning innate human capital, lottery that is repeated for each cohort of newborn persons.

If we denote by $N_{t}$ the number of potential young adults at time $t$ (i.e. abstracting from mortality), that is, the number of births at time $t-1$, the

\footnotetext{
${ }^{6}$ Another difference lies in the fact that Chakraborty (2004) considers the possibility of endogenous survival conditions (depending on public health spendings proportional to the wage), whereas we keep here survival conditions as exogenous (but also related to income).

${ }^{7}$ Our reliance on a three-class model is made for analytical tractability. Section 6 develops a numerical example with 10 income classes.
} 
number of potential young adults of type $i$ at time $t$, denoted by $N_{i t}$, is:

$$
N_{i t}=n_{i} N_{t}
$$

This assumption of constant proportions of the three types of individuals (in potential terms) is made for the sake of analytical tractability. ${ }^{8}$

The wage rate for a young individual of type $i \in\{1,2,3\}$ at time $t$, denoted by $w_{i t}$, is given by:

$$
w_{i t}=w_{t} \lambda_{i}
$$

where $w_{t}$ is the wage rate normalized for human capital, i.e. $w_{t} \equiv \frac{w_{i t}}{\lambda_{i}}$.

The probability to survive the first period (childhood) is denoted by $\phi_{i} \in$ ]0,1]. The probability to survive the second period (young adulthood) conditionally on having survived childhood is denoted by $\left.\left.\pi_{i} \in\right] 0,1\right]$.

We assume a perfect rank correlation between innate human capital and survival probabilities, so that:

$$
\begin{aligned}
& 0<\phi_{1}<\phi_{2}<\phi_{3} \leq 1 \\
& 0<\pi_{1}<\pi_{2}<\pi_{3} \leq 1
\end{aligned}
$$

At this stage, it is crucial to emphasize the different roles played by survival probabilities. Survival in the second period (i.e. probabilities $\phi_{i}$ ) has the effect of determining the size of the labor force. Survival in the third period (i.e. probabilities $\pi_{i}$ ) influences the number of retirees, in particular the number of poor retirees, namely those with the lowest productivity.

\subsection{Production}

The production process involves capital $K_{t}$ and efficient labor $L_{t}$. We assume a production function with constant returns to scale (CRS):

$$
Y_{t}=F\left(K_{t}, L_{t}\right)
$$

where $Y_{t}$ is the output.

Efficient labor $L_{t}$ is defined as the total number of efficiency units at time $t$ :

$$
L_{t} \equiv N_{t} \sum_{i=1,2,3} n_{i} \phi_{i} \lambda_{i}
$$

where $N_{t}=N_{t-1} g$.

Given CRS, we can rewrite this production process in intensive terms as:

$$
y_{t}=f\left(k_{t}\right)
$$

\footnotetext{
${ }^{8}$ This assumption amounts to assume that, independently from the survival conditions faced by the members of the previous cohort, we know for sure that, in the following cohort, the same proportions of the three types will prevail in terms of innate human capital. To see this, take the extreme case where all low productivity individuals die before becoming adults and parents. We then suppose that, among the children of two remaining types, there will be the same proportion of high and low productivity as in the previous cohort.
} 
where $y_{t} \equiv \frac{Y_{t}}{L_{t}}$ and $k_{t} \equiv \frac{K_{t}}{L_{t}}$ denote, respectively, the output per efficient labor unit and the capital stock per efficient labor unit.

It is assumed that the capital stock fully depreciates after one period of use.

Moreover, we assume that factors are paid at their marginal productivity:

$$
\begin{aligned}
& w_{t}=f\left(k_{t}\right)-k f^{\prime}\left(k_{t}\right) \\
& R_{t}=f^{\prime}\left(k_{t}\right)
\end{aligned}
$$

where $R_{t}$ equals one plus the interest rate.

\subsection{Annuities}

Following the literature, we assume that there exists a competitive annuity market, with actuarially fair returns. The raw return factor on savings is thus:

$$
\hat{R}_{i t}=\frac{R_{t}}{\pi_{i}}
$$

We assume here that insurance companies can fully discriminate between the different types of agents, according to their survival chances.

\subsection{Preferences and behavior}

It is assumed that young adults of type 1, i.e. individuals with the lowest innate human capital, do not save resources for their old days, and count on a public pension $b$ for living in the retirement period. ${ }^{9}$ That public pension is funded by a linear $\operatorname{tax} \theta$ on labour income. We thus have:

$$
s_{1 t}=0
$$

Young adults of types 2 and 3 choose a level of saving $s_{i t}$ that maximizes the expected lifetime well-being:

$$
U_{i}=u\left(w_{i t}(1-\theta)-s_{i t}\right)+\pi_{i} u\left(\frac{s_{i t} R_{t+1}}{\pi_{i}}\right)
$$

where $u^{\prime}(\cdot)>0$ and $u^{\prime \prime}(\cdot)<0$. Note that writing the problem this way, we account for the fact that a fraction $\phi_{i}$ of the adults of type $i$ are alive, namely escape early mortality.

The first-order condition yields the standard Euler equation:

$$
u^{\prime}\left(c_{i t}\right)=R_{t+1} u^{\prime}\left(d_{i t+1}\right)
$$

\footnotetext{
${ }^{9}$ Alternatively, we could have assumed that all individuals save a positive amount, with saving propensities varying with the survival probability. This would not have affected our results.
} 


\section{Long-run dynamics}

Given the full depreciation of capital, the total capital stock follows the law:

$$
K_{t+1}=S_{t}
$$

In intensive terms, we have:

$$
g k_{t+1} A=\phi_{2} n_{2} s_{2}\left(R_{t+1}, w_{t}, \theta\right)+\phi_{3} n_{3} s_{3}\left(R_{t+1}, w_{t}, \theta\right)
$$

where $A \equiv \Sigma n_{i} \phi_{i} \lambda_{i}$, while $s_{2}\left(R_{t+1}, w_{t}, \theta\right)$ and $s_{3}\left(R_{t+1}, w_{t}, \theta\right)$ are the savings function for young adults of types 2 and 3 respectively.

At the stationary equilibrium, we have:

$$
g k A=\phi_{2} n_{2} s_{2}(R(k), w(k), \theta)+\phi_{3} n_{3} s_{3}(R(k), w(k), \theta)
$$

As to the revenue constraint, it is:

$$
\theta w\left(\sum_{i=1,2} n_{i} \phi_{i} \lambda_{i}\right)=n_{1} \phi_{1} \pi_{1} b
$$

The existence and uniqueness of a stationary equilibrium depend on the precise shape of savings functions $s_{2}(\cdot)$ and $s_{3}(\cdot)$, and on factor prices $R(k)$ and $w(k)$.

Assuming that preferences are log linear:

$$
u\left(c_{i t}\right)=\log \left(c_{i t}\right)
$$

, and that the production function is a Cobb-Douglas,

$$
y_{t}=B k_{t}^{\alpha}
$$

with $\alpha \in] 0,1[$, we have, for $i=2,3$ :

$$
s_{i t}=\frac{\pi_{i}}{1+\pi_{i}} \lambda_{i}(1-\alpha)(1-\theta) B k_{t}^{\alpha}
$$

Proposition 1 examines the existence, uniqueness and stability of a stationary equilibrium under log linear utility and a Cobb-Douglas technology.

Proposition 1 Consider our economy with a log linear utility function and a Cobb-Douglas production function.

- There exists two stationary equilibria:

$$
k^{-}=0 \text { and } k^{*}=\left[\sum_{i=2,3}\left(\frac{\phi_{i} \pi_{i} n_{i} \lambda_{i}}{1+\pi_{i}}\right) \frac{(1-\alpha)(1-\theta) B}{g A}\right]^{\frac{1}{1-\alpha}}
$$

- $k^{-}$is unstable, and $k^{*}$ is locally stable. 
Proof. See the Appendix.

Hence, the economy admits two stationary equilibria, 0 and $k^{*}$, and only $k^{*}$ is stable. We thus know for sure that our economy will, for any initial conditions $k_{0}>0$, converge towards the stationary equilibrium $k^{*}$. Note that, at the stationary equilibrium $k^{*}$, we have:

$$
w^{*}=B(1-\alpha) k^{* \alpha}=B(1-\alpha)\left[\sum_{i=2,3}\left(\frac{\phi_{i} \pi_{i} n_{i} \lambda_{i}}{1+\pi_{i}}\right) \Omega\right]^{\frac{\alpha}{1-\alpha}}
$$

where $\Omega \equiv \frac{(1-\alpha)(1-\theta) B}{g A}$.

As to the revenue constraint, it is:

$$
\theta w^{*}\left(\Sigma n_{i} \phi_{i} \lambda_{i}\right)=n_{1} \phi_{1} \pi_{1} b^{*}
$$

Proposition 2 states some comparative statics results, concerning the impact of survival conditions on the stationary equilibrium $k^{*}$.

Proposition 2 We have:

$$
\begin{aligned}
\frac{\partial k^{*}}{\partial \phi_{1}} & <0 ; \frac{\partial k^{*}}{\partial \pi_{1}}=0 \\
\frac{\partial k^{*}}{\partial \phi_{i}} & \lessgtr 0 ; \frac{\partial k^{*}}{\partial \pi_{i}}>0 \forall i=2,3 \\
\frac{\partial b^{*}}{\partial \phi_{1}} & <0 ; \frac{\partial b^{*}}{\partial \pi_{1}}<0 \\
\frac{\partial b^{*}}{\partial \phi_{i}} & \lessgtr 0 ; \frac{\partial b^{*}}{\partial \pi_{i}}>0 \forall i=2,3
\end{aligned}
$$

Proof. The proof follows from differentiating $k^{*}$ and $b^{*}$ wrt those arguments.

An improvement in the survival conditions of the low human capital children $\phi_{1}$ reduces the stationary equilibrium capital level. The reason is that a higher proportion of those persons raises the size of the labor force, without affecting capital accumulation (since those persons do not save), so that capital is diluted. On the contrary, a rise in the proportion of low human capital individuals who reach the retirement age $\pi_{1}$ has no effect on the stationary equilibrium, since it does not affect the labour force, nor savings.

For groups $i=2,3$, an improvement in the survival conditions at the young age $\phi_{i}$ has an ambiguous effect on the level of the steady-state capital. Indeed, such an improvement raises the size of the labour force, and, as such, dilutes physical capital. However, at the same time, this raises the proportions of savers in the economy, which pushes towards a higher capital level at the stationary equilibrium. Still for those higher human capital groups, a rise in the probability to reach the old age $\pi_{i}$ has an unambiguous effect on the stationary equilibrium: this contributes to raise $k^{*}$, by increasing the propensity to save through a life horizon effect. 
Finally, it should be stressed that improvements in the survival conditions of the low human capital group reduce, ceteris paribus, the amount of pension they can receive. On the contrary, improvements in the survival conditions of higher human capital groups have an ambiguous effect on the pensions received by the poor, depending on whether the improvement in survival conditions arise at the young age or later on in life. When it arises at the young age, the total impact is ambiguous, since there are more contributors, but with lower wages (due to capital dilution). But when the improvement arises at the old age, the contributions grow thanks to higher wages (through the horizon effect raising capital), and allow the poorest to benefit from higher pensions at the old age.

The various effects mentioned in Proposition 2 are worth being emphasized at this stage, since those effects will be at work when we will consider, in the remaining of this paper, how income-differentiated mortality affects the measurement of poverty in a general equilibrium framework.

\section{The measurement of absolute poverty}

In order to examine how the missing poor phenomenon affects poverty measurement, we will first compute headcount poverty measures at the stationary equilibrium, taking the prevailing differentiated survival conditions as given. Then, we will compute the hypothetical poverty rates that would have prevailed provided there was no income-differentiated mortality. For that purpose, we will consider the hypothetical situation where all individuals face the survival probabilities of the high human capital group (i.e. $\phi_{3}$ and $\pi_{3}$ ). Finally, we will compare the actual and hypothetical measures of poverty, to study how income-differentiated mortality affects measured poverty.

In order to carry out that comparison, we will, throughout the paper, define poverty in terms of available income, that is, income net of taxes to be paid and transfers received. We will first consider a measure of absolute poverty, with a fixed poverty line $\omega>0 .{ }^{10}$

\subsection{Measuring poverty at the young age}

Using the definition of $w^{*}$ at the stable stationary equilibrium, we can write the income level at the young age (net of tax) $y_{y i} \equiv(1-\theta) \lambda_{i} w^{*}$ as:

$$
y_{y i} \equiv(1-\theta) \lambda_{i} B(1-\alpha)\left[\sum_{j=2,3}\left(\frac{\phi_{j} n_{j} \pi_{j} \lambda_{j}}{1+\pi_{j}}\right) \frac{(1-\alpha)(1-\theta) B}{g \sum n_{j} \phi_{j} \lambda_{j}}\right]^{\frac{\alpha}{1-\alpha}}
$$

with $y_{y 1}<y_{y 2}=\frac{\lambda_{2}}{\lambda_{1}} y_{y 1}<y_{y 3}=\frac{\lambda_{3}}{\lambda_{1}} y_{y 1}$. Those income levels can be used to compute the poverty rate at the young age $P_{y}$, i.e. the fraction of the population of young adults whose income net of tax lies below the poverty line $\omega$.

\footnotetext{
${ }^{10}$ The next section will consider the impact of adding prematurely dead persons (i.e. missing persons) on the measurement of relative poverty (with a varying poverty line).
} 
In order to examine the impact of income-differentiated mortality on the measurement of poverty, those poverty rates can be compared with the ones that would prevail in the hypothetical case where there is no income-differentiated mortality. For that purpose, we consider the hypothetical case where all individuals face the survival conditions of the richest (i.e. $\phi_{3}$ and $\pi_{3}$ ). In that hypothetical case, the stationary equilibrium is now:

$$
\hat{k}^{*}=\left[\sum_{i=2,3}\left(\frac{\phi_{3} \pi_{3} n_{i} \lambda_{i}}{1+\pi_{3}}\right) \frac{(1-\alpha)(1-\theta) B}{g \phi_{3} \Sigma n_{j} \lambda_{j}}\right]^{\frac{1}{1-\alpha}}
$$

We thus have, under that hypothetical stationary equilibrium, that the hypothetical income level at the young age for an individual with innate human capital level $i$, denoted by $\hat{y}_{y i}$, is equal to:

$$
\hat{y}_{y i} \equiv(1-\theta) \lambda_{i} B(1-\alpha)\left[\sum_{j=2,3}\left(\frac{\phi_{3} \pi_{3} n_{j} \lambda_{j}}{1+\pi_{3}}\right) \frac{(1-\alpha)(1-\theta) B}{g \phi_{3} \Sigma n_{l} \lambda_{l}}\right]^{\frac{\alpha}{1-\alpha}}
$$

with $\hat{y}_{y 1}<\hat{y}_{y 2}=\frac{\lambda_{2}}{\lambda_{1}} \hat{y}_{y 1}<\hat{y}_{y 3}=\frac{\lambda_{3}}{\lambda_{1}} \hat{y}_{y 1}$.

Those hypothetical income levels at the young age can be used to compute the hypothetical poverty rate at the young age $\hat{P}_{y}$, defined as the fraction of the population of young adults whose income net of tax lies below the poverty line $\omega$ in the hypothetical case where there is no income-differentiated mortality.

Note that those hypothetical income levels at the young age can be either lower or larger than the ones prevailing at the actual stationary equilibrium. To see this, let us compare $y_{y 1}$ with $\hat{y}_{y 1}$. We have:

$$
\hat{y}_{y 1}=y_{y 1}[\Delta]^{\frac{\alpha}{1-\alpha}} \text { where } \Delta \equiv \frac{\sum_{i=2,3}\left(\frac{\phi_{3} \pi_{3} n_{i} \lambda_{i}}{1+\pi_{3}}\right) \frac{1}{\phi_{3} \Sigma n_{j} \lambda_{j}}}{\sum_{i=2,3}\left(\frac{\phi_{i} \pi_{i} n_{i} \lambda_{i}}{1+\pi_{i}}\right) \frac{1}{\Sigma n_{j} \phi_{j} \lambda_{j}}}
$$

From that expression, it follows that $y_{y 1} \gtrless \hat{y}_{y 1}$ if and only if $\Delta \lessgtr 1$. There are three effects at work. The numerator and denominator of $\Delta$ are the sum of two terms, each term being the product of two factors. The second factor reflects the pure capital dilution effect. The higher that effect is, and the more likely is the fact that $y_{y 1}>\hat{y}_{y 1}$. The first factor reflects the impact of changing survival conditions on savings, through (i) the number of savers of the two types; (ii) the horizon effect induced by improving survival conditions from $\phi_{2} \pi_{2}$ to $\phi_{3} \pi_{3}$ for type 2. If the horizon effect is sufficiently strong, then the numerator exceeds the denominator, and this favors the case where $y_{y 1}<\hat{y}_{y 1}$. Similar comparisons can be made for other income classes. Then, one can compare the young age poverty rates under the two income distributions (actual and hypothetical).

Proposition 3 summarizes our results regarding the comparison of the unadjusted and adjusted poverty rates when the capital dilution effects dominates 
other effects. For the sake of presentation, we will, throughout this paper, focus on the case where, before the adjustment, the income group 1 lies in poverty, whereas the income group 3 does not lie in poverty. ${ }^{11}$

Proposition 3 Suppose that the capital dilution effect dominates other effects, that is: $\Delta<1$, so that $y_{y i}>\hat{y}_{y i} \forall i=1,2,3$.

- If $y_{y 1}<\omega \leq \frac{\lambda_{2}}{\lambda_{1}} y_{y 1}$

- if $y_{y 1} \Delta^{\frac{\alpha}{1-\alpha}}<\omega \leq \frac{\lambda_{2}}{\lambda_{1}} y_{y 1} \Delta^{\frac{\alpha}{1-\alpha}}$, then $P_{y}=\frac{\phi_{1} n_{1}}{\Sigma \phi_{i} n_{i}}<\hat{P}_{y}=\frac{n_{1}}{\Sigma n_{i}} ;$

- if $\frac{\lambda_{2}}{\lambda_{1}} y_{y 1} \Delta^{\frac{\alpha}{1-\alpha}}<\omega \leq \frac{\lambda_{3}}{\lambda_{1}} y_{y 1} \Delta^{\frac{\alpha}{1-\alpha}}$, then $P_{y}=\frac{\phi_{1} n_{1}}{\Sigma \phi_{i} n_{i}}<\hat{P}_{y}=\frac{n_{1}+n_{2}}{\Sigma n_{i}}$;

- if $\frac{\lambda_{3}}{\lambda_{1}} y_{y 1} \Delta^{\frac{\alpha}{1-\alpha}}<\omega$, then $P_{y}=\frac{\phi_{1} n_{1}}{\Sigma \phi_{i} n_{i}}<\hat{P}_{y}=1$;

- If $\frac{\lambda_{2}}{\lambda_{1}} y_{y 1}<\omega \leq \frac{\lambda_{3}}{\lambda_{1}} y_{y 1}$

- if $\frac{\lambda_{2}}{\lambda_{1}} y_{y 1} \Delta^{\frac{\alpha}{1-\alpha}}<\omega \leq \frac{\lambda_{3}}{\lambda_{1}} y_{y 1} \Delta^{\frac{\alpha}{1-\alpha}}$, then $P_{y}=\frac{\phi_{1} n_{1}+\phi_{2} n_{2}}{\Sigma \phi_{i} n_{i}}<\hat{P}_{y}=$ $\frac{n_{1}+n_{2}}{\Sigma n_{i}}$;

- if $\frac{\lambda_{3}}{\lambda_{1}} y_{y 1} \Delta^{\frac{\alpha}{1-\alpha}}<\omega$, then $P_{y}=\frac{\phi_{1} n_{1}+\phi_{2} n_{2}}{\Sigma \phi_{i} n_{i}}<\hat{P}_{y}=1$;

Proof. See the Appendix.

Proposition 3 states that, when the capital dilution effect dominates other effects, adding the prematurely dead persons raises poverty measures at the young age. The underlying intuition goes as follows. In that case, the addition of missing persons pushes the incomes of all young individuals down, so that poverty must go up. Thus, in that case, income-differentiated mortality has tended to bias poverty measures downwards, since measured poverty would have been higher provided all individuals had faced the same survival conditions.

Let us now consider the case where the horizon effect dominates other effects, so that the inclusion of missing persons tends to raise the income level of each person. That case is considered in Proposition $4 .^{12}$

Proposition 4 Suppose that the horizon effect dominates other effects, that is, $\Delta>1$, so that $y_{y i}<\hat{y}_{y i} \forall i=1,2,3$.

- If $y_{y 1}<\omega \leq \frac{\lambda_{2}}{\lambda_{1}} y_{y 1}$

- if $y_{y 1} \Delta^{\frac{\alpha}{1-\alpha}}<\omega \leq \frac{\lambda_{2}}{\lambda_{1}} y_{y 1} \Delta^{\frac{\alpha}{1-\alpha}}$, then $P_{y}=\frac{\phi_{1} n_{1}}{\Sigma \phi_{i} n_{i}}<\hat{P}_{y}=\frac{n_{1}}{\Sigma n_{i}}$;

- if $y_{y 1} \Delta^{\frac{\alpha}{1-\alpha}} \geq \omega$, then $P_{y}=\frac{\phi_{1} n_{1}}{\Sigma \phi_{i} n_{i}}>\hat{P}_{y}=0$;

- If $\frac{\lambda_{2}}{\lambda_{1}} y_{y 1}<\omega \leq \frac{\lambda_{3}}{\lambda_{1}} y_{y 1}$

\footnotetext{
${ }^{11}$ See the Appendix for the presentation of all cases, including the ones under either no one is in poverty before the adjustment, or everyone is in poverty before the adjustement.

${ }^{12}$ Here again, we focus on the case where $y_{y 1}<\omega$ and $y_{y 3}>\omega$.
} 


$$
\begin{aligned}
& \text { - if } \frac{\lambda_{2}}{\lambda_{1}} y_{y 1} \Delta^{\frac{\alpha}{1-\alpha}}<\omega \leq \frac{\lambda_{3}}{\lambda_{1}} y_{y 1} \Delta^{\frac{\alpha}{1-\alpha}} \text {, then } P_{y}=\frac{\phi_{1} n_{1}+\phi_{2} n_{2}}{\Sigma \phi_{i} n_{i}}<\hat{P}_{y}= \\
& \quad \frac{n_{1}+n_{2}}{\Sigma n_{i}} ; \\
& \text { - if } y_{y 1} \Delta^{\frac{\alpha}{1-\alpha}}<\omega \leq \frac{\lambda_{2}}{\lambda_{1}} y_{y 1} \Delta^{\frac{\alpha}{1-\alpha}} \text {, then } P_{y}=\frac{\phi_{1} n_{1}+\phi_{2} n_{2}}{\Sigma \phi_{i} n_{i}} \gtrless \hat{P}_{y}= \\
& \quad \frac{n_{1}}{\Sigma n_{i}} \Longleftrightarrow\left(\phi_{1} n_{1}+\phi_{2} n_{2}\right)\left(n_{2}+n_{3}\right) \gtrless \phi_{3} n_{1} n_{3} ; \\
& \text { - if } \omega \leq y_{y 1} \Delta^{\frac{\alpha}{1-\alpha}}<\frac{\lambda_{2}}{\lambda_{1}} y_{y 1} \Delta^{\frac{\alpha}{1-\alpha}} \text {, then } P_{y}=\frac{\phi_{1} n_{1}+\phi_{2} n_{2}}{\Sigma \phi_{i} n_{i}}>\hat{P}_{y}=0 ;
\end{aligned}
$$

Proof. The proof follows the same procedure as for Proposition 3.

When the horizon effect dominates, the income level of each individual in the population is raised, and one may thus expect that the poverty rate falls. But this does not necessarily happen. In two cases, adding missing persons does still raise the measured poverty, despite the general rise in the income of each person. Those cases arise when the rise in the income of the poor is not sufficient to allow these to go beyond the poverty line. Hence, the addition of missing persons, which includes more addition within low human capital groups than among other groups, raises measured poverty, even though general equilibrium effects raised the income of everyone. Thus, when the horizon effect dominates, the impact of income-differentiated mortality on poverty measures is ambiguous.

\subsection{Measuring poverty at the old age}

At the stable stationary equilibrium, the income of the old with innate human capital of type 1 is:

$$
y_{o 1} \equiv b^{*}=\left[\sum_{i=2,3}\left(\frac{\phi_{i} \pi_{i} n_{i} \lambda_{i}}{1+\pi_{i}}\right) \Omega\right]^{\frac{\alpha}{1-\alpha}} \frac{\theta B(1-\alpha)\left(\Sigma n_{j} \phi_{j} \lambda_{j}\right)}{n_{1} \phi_{1} \pi_{1}}
$$

We have also, for $i=2,3$ :

$$
y_{o i} \equiv \frac{R^{*} s_{i}^{*}}{\pi_{i}}=\left[\sum_{j=2,3}\left(\frac{\phi_{j} \pi_{j} n_{j} \lambda_{j}}{1+\pi_{j}}\right) \Omega\right]^{\frac{2 \alpha-1}{1-\alpha}} \frac{\lambda_{i}(1-\alpha)(1-\theta) B^{2} \alpha}{1+\pi_{i}}
$$

Hence we have:

$$
y_{o 2}=\frac{\lambda_{2}}{1+\pi_{2}} \Xi y_{o 1} \text { and } y_{o 3}=\frac{\lambda_{3}}{1+\pi_{3}} \Xi y_{o 1}
$$

where

$$
\Xi \equiv \frac{g n_{1} \phi_{1} \pi_{1} \alpha}{\theta(1-\alpha)\left(\frac{\phi_{2} n_{2} \pi_{2} \lambda_{2}}{1+\pi_{2}}+\frac{\phi_{3} n_{3} \pi_{3} \lambda_{3}}{1+\pi_{3}}\right)}
$$

Based on those income levels, on can compute the old-age poverty rate $P_{o}$.

Here again, this can then be compared with the hypothetical poverty rate $\hat{P}_{o}$ that would have prevailed provided all individuals benefited from the survival 
conditions of the richest. At the hypothetical stationary equilibrium, the income level at the old age for type 1 is:

$$
\hat{y}_{o 1} \equiv\left[\sum_{i=2,3}\left(\frac{\phi_{3} \pi_{3} n_{i} \lambda_{i}}{1+\pi_{3}}\right) \frac{(1-\alpha)(1-\theta) B}{g \phi_{3} \Sigma n_{l} \lambda_{l}}\right]^{\frac{\alpha}{1-\alpha}} \frac{\theta B(1-\alpha)\left(\Sigma n_{l} \lambda_{l}\right)}{n_{1} \pi_{3}}
$$

while it is, for $i=2,3$ :

$$
\hat{y}_{o i} \equiv\left[\sum_{j=2,3}\left(\frac{\phi_{3} \pi_{3} n_{j} \lambda_{j}}{1+\pi_{3}}\right) \frac{(1-\alpha)(1-\theta) B}{g \phi_{3} \Sigma n_{l} \lambda_{l}}\right]^{\frac{2 \alpha-1}{1-\alpha}} \frac{\lambda_{i}(1-\alpha)(1-\theta) B B \alpha}{1+\pi_{3}}
$$

with $\hat{y}_{o 3}=\frac{\lambda_{3}}{\lambda_{2}} \hat{y}_{o 2}>\hat{y}_{o 2}$.

Based on those income levels, it is possible to compute the adjusted old-age poverty $\hat{P}_{o}$, and to compare it with its actual level $P_{o}$. Proposition 5 compares the prevalence of old-age poverty before and after having added missing persons, under the assumption that the capital dilution effect is dominant. ${ }^{13}$

\section{Proposition 5 Define}

$$
\Psi \equiv \frac{n_{1} \phi_{1} \pi_{1} \lambda_{2} \alpha g}{\theta\left(1+\pi_{3}\right)(1-\alpha)\left(\frac{\phi_{2} n_{2} \pi_{2} \lambda_{2}}{1+\pi_{2}}+\frac{\phi_{3} n_{3} \pi_{3} \lambda_{3}}{1+\pi_{3}}\right)}
$$

Suppose that the capital dilution effect dominates other effects, that is:

$$
\Delta^{\frac{\alpha}{1-\alpha}} \frac{\phi_{1} \pi_{1}\left(\sum n_{i} \lambda_{i}\right)}{\pi_{3}\left(\sum n_{i} \phi_{i} \lambda_{i}\right)}<1 ; \Delta^{\frac{2 \alpha-1}{1-\alpha}} \frac{1+\pi_{2}}{1+\pi_{3}}<1 ; \Delta^{\frac{2 \alpha-1}{1-\alpha}}<1
$$

, so that $y_{o i}>\hat{y}_{o i} \forall i=1,2,3$.

- If $y_{o 1}<\omega \leq \frac{\lambda_{2}}{1+\pi_{2}} \Xi y_{o 1}$

- if $y_{o 1} \Delta^{\frac{\alpha}{1-\alpha}} \frac{\phi_{1} \pi_{1}\left(\Sigma n_{i} \lambda_{i}\right)}{\pi_{3}\left(\Sigma n_{i} \phi_{i} \lambda_{i}\right)}<\omega \leq y_{o 1} \Delta^{\frac{2 \alpha-1}{1-\alpha}} \Psi$, then $P_{o}=\frac{\phi_{1} \pi_{1} n_{1}}{\Sigma \pi_{i} \phi_{i} n_{i}}<\hat{P}_{o}=$ $\frac{n_{1}}{\Sigma n_{i}}$

- if $y_{o 1} \Delta^{\frac{2 \alpha-1}{1-\alpha}} \Psi<\omega \leq \frac{\lambda_{3}}{\lambda_{2}} y_{o 1} \Delta^{\frac{2 \alpha-1}{1-\alpha}} \Psi$, then $P_{o}=\frac{\phi_{1} \pi_{1} n_{1}}{\Sigma \pi_{i} \phi_{i} n_{i}}<\hat{P}_{o}=$ $\frac{n_{1}+n_{2}}{\Sigma n_{i}}$

- if $\frac{\lambda_{3}}{\lambda_{2}} y_{o 1} \Delta^{\frac{2 \alpha-1}{1-\alpha}} \Psi<\omega$, then $P_{o}=\frac{\phi_{1} \pi_{1} n_{1}}{\Sigma \pi_{i} \phi_{i} n_{i}}<\hat{P}_{o}=1$;

- If $\frac{\lambda_{2}}{1+\pi_{2}} \Xi y_{o 1}<\omega \leq \frac{\lambda_{3}}{1+\pi_{3}} \Xi y_{o 1}$

- if $y_{o 1} \Delta^{\frac{2 \alpha-1}{1-\alpha}} \Psi<\omega \leq \frac{\lambda_{3}}{\lambda_{2}} y_{o 1} \Delta^{\frac{2 \alpha-1}{1-\alpha}} \Psi$, then $P_{o}=\frac{\phi_{1} \pi_{1} n_{1}+\phi_{2} \pi_{2} n_{2}}{\Sigma \pi_{i} \phi_{i} n_{i}}<$ $\hat{P}_{o}=\frac{n_{1}+n_{2}}{\Sigma n_{i}} ;$

\footnotetext{
${ }^{13}$ We focus on the case where, $y_{o 1}<\omega$ and $y_{o 3}>\omega$. We also assume, without loss of generality, that $\hat{y}_{o 1}<\hat{y}_{o 2}, \hat{y}_{o 3}$.
} 


$$
\text { - if } \frac{\lambda_{3}}{\lambda_{2}} y_{o 1} \Delta^{\frac{2 \alpha-1}{1-\alpha}} \Psi<\omega \text {, then } P_{o}=\frac{\phi_{1} \pi_{1} n_{1}+\phi_{2} \pi_{2} n_{2}}{\Sigma \pi_{i} \phi_{i} n_{i}}<\hat{P}_{o}=1 \text {. }
$$

Proof. See the Appendix.

When the capital dilution effect dominates other effects, the addition of missing persons raises measured poverty at the old age. The impact of incomedifferentiated mortality on the measurement of old-age poverty is thus unambiguous here: it contributed to reduce measured poverty.

On the contrary, when the horizon effect dominates other effects, implying a rise in old-age income, it may be the case that adding missing persons reduces measured poverty. But as for young age poverty, the dominance of the horizon effect is a necessary, but not a sufficient condition for a fall of poverty. ${ }^{14}$

Proposition 6 Suppose that the horizon effect dominates other effects, that is:

$$
\Delta^{\frac{\alpha}{1-\alpha}} \frac{\phi_{1} \pi_{1}\left(\Sigma n_{i} \lambda_{i}\right)}{\pi_{3}\left(\Sigma n_{i} \phi_{i} \lambda_{i}\right)}>1 ; \Delta^{\frac{2 \alpha-1}{1-\alpha}} \frac{1+\pi_{2}}{1+\pi_{3}}>1 ; \Delta^{\frac{2 \alpha-1}{1-\alpha}}>1
$$

, so that $y_{o i}<\hat{y}_{o i} \forall i=1,2,3$.

- If $y_{o 1}<\omega \leq \frac{\lambda_{2}}{1+\pi_{2}} \Xi y_{o 1}$

- if $y_{o 1} \Delta^{\frac{\alpha}{1-\alpha}} \frac{\phi_{1} \pi_{1}\left(\Sigma n_{i} \lambda_{i}\right)}{\pi_{3}\left(\Sigma n_{i} \phi_{i} \lambda_{i}\right)}<\omega \leq y_{o 1} \Delta^{\frac{2 \alpha-1}{1-\alpha}} \Psi$, then $P_{o}=\frac{\phi_{1} \pi_{1} n_{1}}{\Sigma \pi_{i} \phi_{i} n_{i}}<\hat{P}_{o}=$ $\frac{n_{1}}{\Sigma n_{i}}$;

- if $y_{o 1} \Delta^{\frac{\alpha}{1-\alpha}} \frac{\phi_{1} \pi_{1}\left(\Sigma n_{i} \lambda_{i}\right)}{\pi_{3}\left(\Sigma n_{i} \phi_{i} \lambda_{i}\right)} \geq \omega$, then $P_{o}=\frac{\phi_{1} \pi_{1} n_{1}}{\Sigma \pi_{i} \phi_{i} n_{i}}>\hat{P}_{o}=0$;

- If $\frac{\lambda_{2}}{1+\pi_{2}} \Xi y_{o 1}<\omega \leq \frac{\lambda_{3}}{1+\pi_{3}} \Xi y_{o 1}$

- if $y_{o 1} \Delta^{\frac{2 \alpha-1}{1-\alpha}} \Psi<\omega \leq \frac{\lambda_{3}}{\lambda_{2}} y_{o 1} \Delta^{\frac{2 \alpha-1}{1-\alpha}} \Psi$, then $P_{o}=\frac{\phi_{1} \pi_{1} n_{1}+\phi_{2} \pi_{2} n_{2}}{\Sigma \pi_{i} \phi_{i} n_{i}}<$ $\hat{P}_{o}=\frac{n_{1}+n_{2}}{\Sigma n_{i}}$

- if $y_{01} \Delta^{\frac{\alpha}{1-\alpha}} \frac{\phi_{1} \pi_{1}\left(\Sigma n_{i} \lambda_{i}\right)}{\pi_{3}\left(\Sigma n_{i} \phi_{i} \lambda_{i}\right)}<\omega \leq y_{o 1} \Delta^{\frac{2 \alpha-1}{1-\alpha}} \Psi$, then $P_{o}=\frac{\phi_{1} \pi_{1} n_{1}+\phi_{2} \pi_{2} n_{2}}{\Sigma \pi_{i} \phi_{i} n_{i}} \gtrless$ $\hat{P}_{o}=\frac{n_{1}}{\Sigma n_{i}} \Longleftrightarrow\left(\phi_{1} \pi_{1} n_{1}+\phi_{2} \pi_{2} n_{2}\right)\left(n_{2}+n_{3}\right) \gtrless n_{1} \pi_{3} \phi_{3} n_{3} ;$

- if $\omega \leq y_{o 1} \Delta^{\frac{\alpha}{1-\alpha}} \frac{\phi_{1} \pi_{1}\left(\Sigma n_{i} \lambda_{i}\right)}{\pi_{3}\left(\Sigma n_{i} \phi_{i} \lambda_{i}\right)}<y_{o 1} \Delta^{\frac{2 \alpha-1}{1-\alpha}} \Psi$, then $P_{o}=\frac{\phi_{1} \pi_{1} n_{1}+\phi_{2} \pi_{2} n_{2}}{\Sigma \pi_{i} \phi_{i} n_{i}}>$ $\hat{P}_{o}=0$

Proof. The proof follows the same procedure as for Proposition 5.

Proposition 6 states that, when the horizon effect dominates other effects, so that the addition of missing persons raises the income at the old age for all groups, it is not necessarily the case that adding prematurely dead persons reduces measured poverty. It may be the case that poverty goes up despite favorable general equilibrium effects. This result arises when the growth in individual income is insufficient to allow some previously poor people to reach an income level that exceeds the poverty line. However, since the adjustment

${ }^{14}$ Here again, we focus on the case where, $y_{o 1}<\omega$ and $y_{o 3}>\omega$. 
amounts to add relatively more people in low income groups than in high income groups, we may still have more poverty after the adjustment, despite a dominant horizon effect leading to a rise in everyone's income.

All in all, income-differentiated mortality can have varying effects on measured poverty. When the capital dilution effect dominates (leading to a fall in income for all when the missing persons are added), the income/mortality gradient reduces measured poverty. However, when the horizon effect dominates (leading to a rise in income for all when the missing persons are added), income-differentiated mortality does not necessarily raise measured poverty.

\section{The measurement of relative poverty}

When the poverty line is relative to the income distribution under study, the inclusion of prematurely dead persons has an additional effect, which is not present in the case of measures of absolute poverty: adding missing persons can here affect the level of the relative poverty line, unlike what prevailed so far.

In order to examine how income-differentiated mortality affects the measurement of relative poverty, we will, as above, distinguish between poverty at the young age and at the old age. The relative poverty line being usually defined as a proportion of median income of the population considered, we will fix it at a proportion $0<x<1$ of the median age-specific income.

\subsection{Measuring poverty at the young age}

The measure of relative poverty at the young age, denoted by $p_{y}$, can take various values, depending on where the median income $y_{y m}$ lies with respect to income levels $y_{y 1}, y_{y 2}$ and $y_{y 3} \cdot{ }^{15}$ The precise position of the median income depends on the levels of $n_{1}, n_{2}$ and $n_{3}$. If, for instance, we have $n_{2}>>n_{1}, n_{3}$, then the median income is $y_{y m}=y_{y 2}$. The position of $y_{y m}$ depends also on survival probabilities $\phi_{1}, \phi_{2}$ and $\phi_{3}$. When there is a high mortality only among the poorest, that is, when $\phi_{1}$ is very low, the median income is generally equal to $y_{y 2}$. But if all groups except group 3 face high mortality, we have $y_{y m}=y_{y 3}$.

When considering the economy after the inclusion of prematurely dead persons, there are also several possible cases, depending on where the median (adjusted) income $\hat{y}_{y m}$ lies with respect to $\hat{y}_{y 1}, \hat{y}_{y 2}$ and $\hat{y}_{y 3}$. Note that the median income after the addition of missing persons may differ from its level before the adjustment, implying a possible shift of the poverty line.

In order to study the impact of adding prematurely dead persons on the measurement of poverty, we need, as above, to distinguish between the cases where the inclusion of missing persons reduces or raises the income of everyone. Proposition 7 considers the case where the capital dilution effect is dominant. ${ }^{16}$

\footnotetext{
${ }^{15}$ See the Proof of Proposition 7 in the Appendix.

${ }^{16}$ Without loss of generality, we focus on the case where, before the adjustment, the income group 1 lies in poverty (i.e. $y_{y 1}<y_{y m}$ ), whereas the income group 3 does not lie in poverty (i.e. $\left.y_{y m}<y_{y 3}\right)$.
} 
Proposition 7 Suppose that the capital dilution effect dominates other effects, that is: $\Delta<1$, so that $y_{y i}>\hat{y}_{y i} \forall i=1,2,3$. Suppose that $n_{1}, n_{2}$ and $n_{3}$ are such that, after adjustment, the median income remains either in the same group as before, or shifts down to a lower income group.

- If $y_{y m}=y_{y 2}$ and $1<x \frac{\lambda_{2}}{\lambda_{1}}$,

- if $\hat{y}_{y m}=\hat{y}_{y 1}$, then $p_{y}=\frac{\phi_{1} n_{1}}{\Sigma \phi_{i} n_{i}}>\hat{p}_{y}=0$;

- if $\hat{y}_{y m}=\hat{y}_{y 2}$ and given $1<x \frac{\lambda_{2}}{\lambda_{1}}$, then $p_{y}=\frac{\phi_{1} n_{1}}{\Sigma \phi_{i} n_{i}}<\hat{p}_{y}=\frac{n_{1}}{\Sigma n_{i}}$;

- If $y_{y m}=y_{y 3}$ and $1<x \frac{\lambda_{3}}{\lambda_{1}} \leq \frac{\lambda_{2}}{\lambda_{1}}$,

- if $\hat{y}_{y m}=\hat{y}_{y 1}$, then $p_{y}=\frac{\phi_{1} n_{1}}{\Sigma \phi_{i} n_{i}}>\hat{p}_{y}=0$;

- if $\hat{y}_{y m}=\hat{y}_{y 2}$ and $1 \geq x \frac{\lambda_{2}}{\lambda_{1}}$, then $p_{y}=\frac{\phi_{1} n_{1}}{\Sigma \phi_{i} n_{i}}>\hat{p}_{y}=0$;

- if $\hat{y}_{y m}=\hat{y}_{y 2}$ and $1<x \frac{\lambda_{2}}{\lambda_{1}}$, then $p_{y}=\frac{\phi_{1} n_{1}}{\Sigma \phi_{i} n_{i}}<\hat{p}_{y}=\frac{n_{1}}{\Sigma n_{i}}$;

- if $\hat{y}_{y m}=\hat{y}_{y 3}$ and $1<x \frac{\lambda_{3}}{\lambda_{1}} \leq \frac{\lambda_{2}}{\lambda_{1}}$, then $p_{y}=\frac{\phi_{1} n_{1}}{\Sigma \phi_{i} n_{i}}<\hat{p}_{y}=\frac{n_{1}}{\Sigma n_{i}}$;

- If $y_{y m}=y_{y 3}$ and $1<\frac{\lambda_{2}}{\lambda_{1}}<x \frac{\lambda_{3}}{\lambda_{1}}$,

- if $\hat{y}_{y m}=\hat{y}_{y 1}$, then $p_{y}=\frac{\phi_{1} n_{1}+\phi_{2} n_{2}}{\Sigma \phi_{i} n_{i}}>\hat{p}_{y}=0$;

- if $\hat{y}_{y m}=\hat{y}_{y 2}$ and $1 \geq x \frac{\lambda_{2}}{\lambda_{1}}$, then $p_{y}=\frac{\phi_{1} n_{1}+\phi_{2} n_{2}}{\Sigma \phi_{i} n_{i}}>\hat{p}_{y}=0$;

- if $\hat{y}_{y m}=\hat{y}_{y 2}$ and $1<x \frac{\lambda_{2}}{\lambda_{1}}$, then $p_{y}=\frac{\phi_{1} n_{1}+\phi_{2} n_{2}}{\Sigma \phi_{i} n_{i}} \gtrless \hat{p}_{y}=\frac{n_{1}}{\Sigma n_{i}} \Longleftrightarrow$ $\left(\phi_{1} n_{1}+\phi_{2} n_{2}\right)\left(n_{2}+n_{3}\right) \gtrless \phi_{3} n_{1} n_{3} ;$

- if $\hat{y}_{y m}=\hat{y}_{y 3}$ and $1<\frac{\lambda_{2}}{\lambda_{1}}<x \frac{\lambda_{3}}{\lambda_{1}}$, then $p_{y}=\frac{\phi_{1} n_{1}+\phi_{2} n_{2}}{\Sigma \phi_{i} n_{i}}<\hat{p}_{y}=\frac{n_{1}+n_{2}}{\Sigma n_{i}}$.

Proof. See the Appendix.

Unlike under absolute poverty measurement, the addition of prematurely dead persons, when it leads to a fall in everyone's income, can have various effects on the measurement of poverty. Under absolute poverty measurement, adding missing persons contributed to raise measured poverty when capital dilution dominates. This is not necessarily the case here, since the addition of missing persons can push the poverty line down, and make individuals escape from relative poverty. Thus, unlike under absolute poverty, income-differentiated mortality has an ambiguous effect on the measurement of relative poverty.

Another interesting difference with respect to the case of absolute poverty is the fact that the conditions mentioned in Proposition 7 do not depend on the relative size of the income fall due to the addition of missing persons, that is, these conditions do not depend on how large $\Delta$ is, unlike what prevailed under absolute poverty. The underlying intuition is that all income levels - including the relative poverty line - are here multiplied by a common factor $\Delta^{\frac{\alpha}{1-\alpha}}$. Hence, the size of $\Delta$ is neutral concerning the impact of adding missing persons. 
The key factors determining the impact of adding prematurely dead persons are the differentials in productivity levels $\lambda_{i}$ and the parameter $x$. To interpret Proposition 7, let us take the case where the median income $y_{y m}$ is $y_{y 2}$. Supposing that there exists initially some poverty (i.e. $1<x \frac{\lambda_{2}}{\lambda_{1}}$ ), two opposite cases can arise. If the addition of missing persons makes the poverty line fall to the income of the first group, then the extent of measured poverty falls because of the adjustment. If, however, the poverty line remains related to the second group, then the extent of measured poverty goes up with the addition of missing persons (whose additions are relatively larger in group 1 ).

Proposition 8 considers the case where the horizon effect dominates other effects, and leads to a general rise in all income levels. In that case, the median income is likely to grow towards the income level of a higher group. ${ }^{17}$

Proposition 8 Suppose that the horizon effect dominates other effects, that is: $\Delta>1$, so that $y_{y i}<\hat{y}_{y i} \forall i=1,2,3$. Suppose that $n_{1}, n_{2}$ and $n_{3}$ are such that, after adjustment, the median income remains either in the same group as before, or shifts up to a higher income group.

- If $y_{y m}=y_{y 2}$ and $1<x \frac{\lambda_{2}}{\lambda_{1}}$,

- if $\hat{y}_{y m}=\hat{y}_{y 2}$ and $1<x \frac{\lambda_{2}}{\lambda_{1}}$, then $p_{y}=\frac{\phi_{1} n_{1}}{\Sigma \phi_{i} n_{i}}<\hat{p}_{y}=\frac{n_{1}}{\Sigma n_{i}}$;

- if $\hat{y}_{y m}=\hat{y}_{y 3}$ and $1<x \frac{\lambda_{3}}{\lambda_{1}} \leq \frac{\lambda_{2}}{\lambda_{1}}$, then $p_{y}=\frac{\phi_{1} n_{1}}{\Sigma \phi_{i} n_{i}}<\hat{p}_{y}=\frac{n_{1}}{\Sigma n_{i}}$;

- if $\hat{y}_{y m}=\hat{y}_{y 3}$ and $1<\frac{\lambda_{2}}{\lambda_{1}}<x \frac{\lambda_{3}}{\lambda_{1}}$, then $p_{y}=\frac{\phi_{1} n_{1}}{\Sigma \phi_{i} n_{i}}<\hat{p}_{y}=\frac{n_{1}+n_{2}}{\Sigma n_{i}}$;

- If $y_{y m}=y_{y 3}$ and $1<x \frac{\lambda_{3}}{\lambda_{1}} \leq \frac{\lambda_{2}}{\lambda_{1}}$, then $\hat{y}_{y m}=\hat{y}_{y 3}$, so that $p_{y}=\frac{\phi_{1} n_{1}}{\Sigma \phi_{i} n_{i}}<$ $\hat{p}_{y}=\frac{n_{1}}{\Sigma n_{i}}$;

- If $y_{y m}=y_{y 3}$ and $1<\frac{\lambda_{2}}{\lambda_{1}}<x \frac{\lambda_{3}}{\lambda_{1}}$, then $\hat{y}_{y m}=\hat{y}_{y 3}$, so that $p_{y}=\frac{\phi_{1} n_{1}+\phi_{2} n_{2}}{\Sigma \phi_{i} n_{i}}<$ $\hat{p}_{y}=\frac{n_{1}+n_{2}}{\Sigma n_{i}}$.

Proof. The proof follows the same procedure as for Proposition 7 .

When the horizon effect dominates, so that the median income remains either in the same group as before, or shifts down to a higher income group, the addition of missing persons raises measured poverty. This result is different from what prevailed under absolute poverty measurement, where, in the same conditions, it was possible that the addition of missing poor persons reduces measured poverty. This case is not possible here. The intuition goes as follows. First, if the rise in individual incomes is sufficiently low, so that the poverty line remains related to the same income group as initially, then the mere fact that the addition of missing persons concerns mainly the ones of group 1 suffices to raise measured poverty. If, on the contrary, the rise in incomes is sufficiently large, this raises the poverty line, which pushes more persons into poverty.

${ }^{17}$ Here again, we assume $y_{y 1}<y_{y m}$ and $y_{y m}<y_{y 3}$. 
In sum, the addition of prematurely dead persons has quite different consequences on the measurement of poverty at the young age, depending on whether we consider measures of absolute or relative poverty. When the capital dilution effects dominates other effects, measures of absolute poverty necessarily go up when missing persons are added, whereas measures of relative poverty may go up or down, depending on the movement of the poverty line. But when the horizon effect dominates other effects, measures of absolute poverty may increase or decrease, whereas measures of relative poverty necessarily increase.

\subsection{Measuring poverty at the old age}

Proposition 9 compares measures of old-age relative poverty before and after the addition of prematurely dead persons (i.e. $p_{o} \lessgtr \hat{p}_{o}$ ). ${ }^{18}$

Proposition 9 Suppose that the capital dilution effect dominates other effects, that is:

$$
\Delta^{\frac{\alpha}{1-\alpha}} \frac{\phi_{1} \pi_{1}\left(\Sigma n_{i} \lambda_{i}\right)}{\pi_{3}\left(\sum n_{i} \phi_{i} \lambda_{i}\right)}<1 ; \Delta^{\frac{2 \alpha-1}{1-\alpha}} \frac{1+\pi_{2}}{1+\pi_{3}}<1 ; \Delta^{\frac{2 \alpha-1}{1-\alpha}}<1
$$

, so that $y_{o i}>\hat{y}_{o i} \forall i=1,2,3$. Suppose that $n_{1}, n_{2}$ and $n_{3}$ are such that, after adjustment, the median income remains either in the same group as before, or shifts down to a lower income group.

- If $y_{o m}=y_{o 2}$ and $1<x \frac{\lambda_{2}}{1+\pi_{2}} \Xi$,

- if $\hat{y}_{o m}=\hat{y}_{o 1}$, then $p_{o}=\frac{\pi_{1} \phi_{1} n_{1}}{\Sigma \phi_{i} \pi_{i} n_{i}}>\hat{p}_{o}=0$;

- if $\hat{y}_{o m}=\hat{y}_{o 2}$ and if $\frac{\theta(1-\alpha)}{g} \geq \frac{x n_{1} \lambda_{2} \alpha}{n_{2} \lambda_{2}+n_{3} \lambda_{3}}$, then $p_{o}=\frac{\pi_{1} \phi_{1} n_{1}}{\Sigma \phi_{i} \pi_{i} n_{i}}>\hat{p}_{o}=0$;

- if $\hat{y}_{o m}=\hat{y}_{o 2}$ and if $\frac{\theta(1-\alpha)}{g}<\frac{x n_{1} \lambda_{2} \alpha}{n_{2} \lambda_{2}+n_{3} \lambda_{3}} \leq \frac{n_{1} \lambda_{3} \alpha}{n_{2} \lambda_{2}+n_{3} \lambda_{3}}$, then $p_{o}=$ $\frac{\pi_{1} \phi_{1} n_{1}}{\Sigma \phi_{i} \pi_{i} n_{i}}<\hat{p}_{O}=\frac{n_{1}}{\Sigma n_{i}}$

- If $y_{o m}=y_{o 3}$ and $1<x \frac{\lambda_{3}}{1+\pi_{3}} \Xi \leq \frac{\lambda_{2}}{1+\pi_{2}} \Xi$,

- if $\hat{y}_{o m}=\hat{y}_{o 1}$, then $p_{o}=\frac{\pi_{1} \phi_{1} n_{1}}{\Sigma \phi_{i} \pi_{i} n_{i}}>\hat{p}_{o}=0$;

- if $\hat{y}_{o m}=\hat{y}_{o 2}$ and $\frac{\theta(1-\alpha)}{g} \geq \frac{x n_{1} \lambda_{2} \alpha}{n_{2} \lambda_{2}+n_{3} \lambda_{3}}$, then $p_{o}=\frac{\pi_{1} \phi_{1} n_{1}}{\Sigma \phi_{i} \pi_{i} n_{i}}>\hat{p}_{o}=0$;

- if $\hat{y}_{o m}=\hat{y}_{o 2}$ and $\frac{\theta(1-\alpha)}{g}<\frac{x n_{1} \lambda_{2} \alpha}{n_{2} \lambda_{2}+n_{3} \lambda_{3}} \leq \frac{n_{1} \lambda_{3} \alpha}{n_{2} \lambda_{2}+n_{3} \lambda_{3}}$, then $p_{o}=$ $\frac{\pi_{1} \phi_{1} n_{1}}{\Sigma \phi_{i} \pi_{i} n_{i}}<\hat{p}_{O}=\frac{n_{1}}{\Sigma n_{i}}$

- if $\hat{y}_{o m}=\hat{y}_{o 3}$ and $\frac{\theta(1-\alpha)}{g} \geq \frac{x n_{1} \lambda_{3} \alpha}{n_{2} \lambda_{2}+n_{3} \lambda_{3}}$, then $p_{o}=\frac{\pi_{1} \phi_{1} n_{1}}{\Sigma \phi_{i} \pi_{i} n_{i}}>\hat{p}_{o}=0$;

- if $\hat{y}_{o m}=\hat{y}_{o 3}$ and $\frac{\theta(1-\alpha)}{g}<\frac{x n_{1} \lambda_{3} \alpha}{n_{2} \lambda_{2}+n_{3} \lambda_{3}} \leq \frac{n_{1} \lambda_{2} \alpha}{n_{2} \lambda_{2}+n_{3} \lambda_{3}}$, then $p_{o}=$ $\frac{\pi_{1} \phi_{1} n_{1}}{\Sigma \phi_{i} \pi_{i} n_{i}}<\hat{p}_{O}=\frac{n_{1}}{\Sigma n_{i}}$

${ }^{18}$ Here again, we assume $y_{o 1}<y_{o m}$ and $y_{o m}<y_{o 3}$. 
$\begin{aligned}- \text { if } \hat{y}_{o m} & =\hat{y}_{o 3} \text { and } \frac{\theta(1-\alpha)}{g}<\frac{n_{1} \lambda_{2} \alpha}{n_{2} \lambda_{2}+n_{3} \lambda_{3}}<\frac{x n_{1} \lambda_{3} \alpha}{n_{2} \lambda_{2}+n_{3} \lambda_{3}} \text {, then } p_{o}= \\ \frac{\pi_{1} \phi_{1} n_{1}}{\Sigma \phi_{i} n_{i}} & <\hat{p}_{o}=\frac{n_{1}+n_{2}}{\Sigma n_{2}} \text {. }\end{aligned}$ $\frac{\pi_{1} \phi_{1} n_{1}}{\Sigma \phi_{i} \pi_{i} n_{i}}<\hat{p}_{O}=\frac{n_{1}+n_{2}}{\Sigma n_{i}}$

- If $y_{o m}=y_{o 3}$ and $1<\frac{\lambda_{2}}{1+\pi_{2}} \Xi<x \frac{\lambda_{3}}{1+\pi_{3}} \Xi$,

- if $\hat{y}_{o m}=\hat{y}_{o 1}$, then $p_{o}=\frac{\pi_{1} \phi_{1} n_{1}+\pi_{2} \phi_{2} n_{2}}{\Sigma \phi_{i} \pi_{i} n_{i}}>\hat{p}_{o}=0$;

- if $\hat{y}_{o m}=\hat{y}_{o 2}$ and $\frac{\theta(1-\alpha)}{g} \geq \frac{x n_{1} \lambda_{2} \alpha}{n_{2} \lambda_{2}+n_{3} \lambda_{3}}$, then $p_{o}=\frac{\pi_{1} \phi_{1} n_{1}+\pi_{2} \phi_{2} n_{2}}{\Sigma \phi_{i} \pi_{i} n_{i}}>$ $\hat{p}_{o}=0$;

- if $\hat{y}_{o m}=\hat{y}_{o 2}$ and $\frac{\theta(1-\alpha)}{g}<\frac{x n_{1} \lambda_{2} \alpha}{n_{2} \lambda_{2}+n_{3} \lambda_{3}} \leq \frac{n_{1} \lambda_{3} \alpha}{n_{2} \lambda_{2}+n_{3} \lambda_{3}}$, then $p_{o}=$ $\frac{\pi_{1} \phi_{1} n_{1}+\pi_{2} \phi_{2} n_{2}}{\Sigma \phi_{i} \pi_{i} n_{i}} \gtrless \hat{p}_{o}=\frac{n_{1}}{\Sigma n_{i}} \Longleftrightarrow\left(\phi_{1} \pi_{1} n_{1}+\phi_{2} \pi_{2} n_{2}\right)\left(n_{2}+n_{3}\right) \gtrless$ $n_{1} \pi_{3} \phi_{3} n_{3}$

- if $\hat{y}_{o m}=\hat{y}_{o 3}$ and $\frac{\theta(1-\alpha)}{g} \geq \frac{x n_{1} \lambda_{3} \alpha}{n_{2} \lambda_{2}+n_{3} \lambda_{3}}$, then $p_{o}=\frac{\pi_{1} \phi_{1} n_{1}+\pi_{2} \phi_{2} n_{2}}{\Sigma \phi_{i} \pi_{i} n_{i}}>$ $\hat{p}_{o}=0$.

- if $\hat{y}_{o m}=\hat{y}_{o 3}$ and $\frac{\theta(1-\alpha)}{g}<\frac{x n_{1} \lambda_{3} \alpha}{n_{2} \lambda_{2}+n_{3} \lambda_{3}} \leq \frac{n_{1} \lambda_{2} \alpha}{n_{2} \lambda_{2}+n_{3} \lambda_{3}}$, then $p_{o}=$ $\frac{\pi_{1} \phi_{1} n_{1}+\pi_{2} \phi_{2} n_{2}}{\Sigma \phi_{i} \pi_{i} n_{i}} \gtrless \hat{p}_{o}=\frac{n_{1}}{\Sigma n_{i}} \Longleftrightarrow\left(\phi_{1} \pi_{1} n_{1}+\phi_{2} \pi_{2} n_{2}\right)\left(n_{2}+n_{3}\right) \gtrless$ $n_{1} \pi_{3} \phi_{3} n_{3}$;

- if $\hat{y}_{o m}=\hat{y}_{o 3}$ and $\frac{\theta(1-\alpha)}{g}<\frac{n_{1} \lambda_{2} \alpha}{n_{2} \lambda_{2}+n_{3} \lambda_{3}}<\frac{x n_{1} \lambda_{3} \alpha}{n_{2} \lambda_{2}+n_{3} \lambda_{3}}$, then $p_{o}=$ $\frac{\pi_{1} \phi_{1} n_{1}+\pi_{2} \phi_{2} n_{2}}{\Sigma \phi_{i} \pi_{i} n_{i}}<\hat{p}_{O}=\frac{n_{1}+n_{2}}{\Sigma n_{i}}$

Proof. See the Appendix.

When the capital dilution effect dominates other effects, the effect of adding prematurely dead persons on the measurement of old age poverty is ambiguous once the poverty line is varying. This result is, again, quite different from what was prevailing under absolute poverty measurement, where the effect was to raise the extent of measured poverty.

To interpret that result, let us focus on the case where the median income at the old age is $y_{o 2}$. If there is initially some old age poverty, it is possible to see a decline in poverty when the adjustment pushes the poverty line down (by reducing the median income to $y_{o 1}$ ). But if the poverty line remains equal to $x y_{o 2}$, it is possible to see a fall or a rise in the prevalence of poverty. Here again, two effects are at work. The poverty line falls when $y_{o 2}$ decreases. Hence individuals of group 1 may possibly escape from poverty. But since $y_{o 1}$ is also falling, it is possible that these remain in poverty, but are now relatively more numerous due to correction for mortality differentials.

Finally, Proposition 10 considers the case where the horizon effect dominates, implying that the inclusion missing persons pushes everyone's income up. ${ }^{19}$

Proposition 10 Suppose that the horizon effect dominates other effects, that is:

$$
\Delta^{\frac{\alpha}{1-\alpha}} \frac{\phi_{1} \pi_{1}\left(\sum n_{i} \lambda_{i}\right)}{\pi_{3}\left(\sum n_{i} \phi_{i} \lambda_{i}\right)}>1 ; \Delta^{\frac{2 \alpha-1}{1-\alpha}} \frac{1+\pi_{2}}{1+\pi_{3}}>1 ; \Delta^{\frac{2 \alpha-1}{1-\alpha}}>1
$$

${ }^{19}$ Here again, we assume $y_{o 1}<y_{o m}$ and $y_{o m}<y_{o 3}$. 
, so that $y_{o i}<\hat{y}_{o i} \forall i=1,2,3$. Suppose that $n_{1}, n_{2}$ and $n_{3}$ are such that, after adjustment, the median income remains either in the same group as before, or shifts up to a higher income group.

- If $y_{o m}=y_{o 2}$ and $1<x \frac{\lambda_{2}}{1+\pi_{2}} \Xi$,

- if $\hat{y}_{o m}=\hat{y}_{o 2}$ and $\frac{\theta(1-\alpha)}{g} \geq \frac{x n_{1} \lambda_{2} \alpha}{n_{2} \lambda_{2}+n_{3} \lambda_{3}}$, then $p_{o}=\frac{\pi_{1} \phi_{1} n_{1}}{\Sigma \phi_{i} \pi_{i} n_{i}}>\hat{p}_{o}=0$;

- if $\hat{y}_{o m}=\hat{y}_{o 2}$ and $\frac{\theta(1-\alpha)}{g}<\frac{x n_{1} \lambda_{2} \alpha}{n_{2} \lambda_{2}+n_{3} \lambda_{3}}$, then $p_{o}=\frac{\pi_{1} \phi_{1} n_{1}}{\Sigma \phi_{i} \pi_{i} n_{i}}<\hat{p}_{o}=\frac{n_{1}}{\Sigma n_{i}}$;

- if $\hat{y}_{o m}=\hat{y}_{o 3}$ and $\frac{\theta(1-\alpha)}{g} \geq \frac{x n_{1} \lambda_{3} \alpha}{n_{2} \lambda_{2}+n_{3} \lambda_{3}}$, then $p_{o}=\frac{\pi_{1} \phi_{1} n_{1}}{\Sigma \phi_{i} \pi_{i} n_{i}}>\hat{p}_{o}=0$;

- if $\hat{y}_{o m}=\hat{y}_{o 3}$ and $\frac{\theta(1-\alpha)}{g}<\frac{x n_{1} \lambda_{3} \alpha}{n_{2} \lambda_{2}+n_{3} \lambda_{3}} \leq \frac{n_{1} \lambda_{2} \alpha}{n_{2} \lambda_{2}+n_{3} \lambda_{3}}$, then $p_{o}=$ $\frac{\pi_{1} \phi_{1} n_{1}}{\Sigma \phi_{i} \pi_{i} n_{i}}<\hat{p}_{o}=\frac{n_{1}}{\Sigma n_{i}}$

- if $\hat{y}_{o m}=\hat{y}_{o 3}$ and $\frac{\theta(1-\alpha)}{g}<\frac{n_{1} \lambda_{2} \alpha}{n_{2} \lambda_{2}+n_{3} \lambda_{3}}<\frac{x n_{1} \lambda_{3} \alpha}{n_{2} \lambda_{2}+n_{3} \lambda_{3}}$, then $p_{o}=$ $\frac{\pi_{1} \phi_{1} n_{1}}{\Sigma \phi_{i} \pi_{i} n_{i}}<\hat{p}_{o}=\frac{n_{1}+n_{2}}{\Sigma n_{i}}$

- If $y_{o m}=y_{o 3}$ and $1<x \frac{\lambda_{3}}{1+\pi_{3}} \Xi \leq \frac{\lambda_{2}}{1+\pi_{2}} \Xi$,

- if $\hat{y}_{o m}=\hat{y}_{o 3}$ and $\frac{\theta(1-\alpha)}{g} \geq \frac{x n_{1} \lambda_{3} \alpha}{n_{2} \lambda_{2}+n_{3} \lambda_{3}}$, then $p_{o}=\frac{\pi_{1} \phi_{1} n_{1}}{\Sigma \phi_{i} \pi_{i} n_{i}}>\hat{p}_{o}=0$;

- if $\hat{y}_{o m}=\hat{y}_{o 3}$ and $\frac{\theta(1-\alpha)}{g}<\frac{x n_{1} \lambda_{3} \alpha}{n_{2} \lambda_{2}+n_{3} \lambda_{3}} \leq \frac{n_{1} \lambda_{2} \alpha}{n_{2} \lambda_{2}+n_{3} \lambda_{3}}$, then $p_{o}=$ $\frac{\pi_{1} \phi_{1} n_{1}}{\Sigma \phi_{i} \pi_{i} n_{i}}<\hat{p}_{o}=\frac{n_{1}}{\Sigma n_{i}} ;$

- if $\hat{y}_{o m}=\hat{y}_{o 3}$ and $\frac{\theta(1-\alpha)}{g}<\frac{n_{1} \lambda_{2} \alpha}{n_{2} \lambda_{2}+n_{3} \lambda_{3}}<\frac{x n_{1} \lambda_{3} \alpha}{n_{2} \lambda_{2}+n_{3} \lambda_{3}}$, then $p_{o}=$ $\frac{\pi_{1} \phi_{1} n_{1}}{\Sigma \phi_{i} \pi_{i} n_{i}}<\hat{p}_{o}=\frac{n_{1}+n_{2}}{\Sigma n_{i}}$

- If $y_{o m}=y_{o 3}$ and $1<\frac{\lambda_{2}}{1+\pi_{2}} \Xi<x \frac{\lambda_{3}}{1+\pi_{3}} \Xi$,

- if $\hat{y}_{o m}=\hat{y}_{o 3}$ and $\frac{\theta(1-\alpha)}{g} \geq \frac{x n_{1} \lambda_{3} \alpha}{n_{2} \lambda_{2}+n_{3} \lambda_{3}}$, then $p_{o}=\frac{\pi_{1} \phi_{1} n_{1}+\phi_{2} \pi_{2} n_{2}}{\Sigma \phi_{i} \pi_{i} n_{i}}>$ $\hat{p}_{o}=0$;

- if $\hat{y}_{o m}=\hat{y}_{o 3}$ and $\frac{\theta(1-\alpha)}{\pi_{3} g}<\frac{x n_{1} \lambda_{3} \alpha}{n_{2} \lambda_{2}+n_{3} \lambda_{3}} \leq \frac{n_{1} \lambda_{2} \alpha}{n_{2} \lambda_{2}+n_{3} \lambda_{3}}$, then $p_{o}=$ $\frac{\pi_{1} \phi_{1} n_{1}+\phi_{2} \pi_{2} n_{2}}{\Sigma \phi_{i} \pi_{i} n_{i}} \gtrless \hat{p}_{o}=\frac{n_{1}}{\Sigma n_{i}} \Longleftrightarrow\left(\phi_{1} \pi_{1} n_{1}+\phi_{2} \pi_{2} n_{2}\right)\left(n_{2}+n_{3}\right) \gtrless$ $n_{1} \pi_{3} \phi_{3} n_{3} ;$

- if $\hat{y}_{o m}=\hat{y}_{o 3}$ and $\frac{\theta(1-\alpha)}{g}<\frac{n_{1} \lambda_{2} \alpha}{n_{2} \lambda_{2}+n_{3} \lambda_{3}}<\frac{x n_{1} \lambda_{3} \alpha}{n_{2} \lambda_{2}+n_{3} \lambda_{3}}$, then $p_{o}=$ $\frac{\pi_{1} \phi_{1} n_{1}+\phi_{2} \pi_{2} n_{2}}{\Sigma \phi_{i} \pi_{i} n_{i}}<\hat{p}_{o}=\frac{n_{1}+n_{2}}{\Sigma n_{i}} ;$

Proof. The proof follows the same procedure as for Proposition 9.

When the horizon effect dominates other effects, it is not necessarily the case that the inclusion of missing persons will reduce old-age poverty. In particular, if the rise in everyone's income has the consequence of making the median income at the old age shift from a low human capital group to a higher human capital group, it is possible, despite the general rise in income levels, that the measured poverty goes up. 
In comparison with absolute poverty (Proposition 6), the contrast is not that strong, since under absolute poverty it was not certain that adding missing persons would, under a dominant horizon effect, suffice to reduce old-age poverty. But the additional ingredient here, which makes that case even less likely than under absolute poverty measurement, is the fact that the poverty line can here go up, and generate extra (relative) poor persons.

\subsection{A summary}

Shifting from measures of absolute poverty to measures of relative poverty complicates the picture, because, for the latter, the addition of prematurely dead persons also modifies the poverty line. Table 1 summarizes our results.

\begin{tabular}{|l|c|c|c|c|}
\hline \hline & \multicolumn{2}{|c|}{ absolute poverty } & \multicolumn{2}{|c|}{ relative poverty } \\
& young & old & young & old \\
capital dilution dominates & $\uparrow$ & $\uparrow$ & $?$ & $?$ \\
horizon effect dominates & $?$ & $?$ & $\uparrow$ & $?$ \\
\hline \hline
\end{tabular}

Table 1: Summary of results

Under absolute poverty measures, it is clear that, when the capital dilution effects dominates, adding prematurely dead persons raises measured poverty. However, once the horizon effect dominates, the effect of adding missing persons is indeterminate. Two effects are at work: first, variations in individual income levels, which can make individuals go above or below the poverty line; second, the addition of missing persons involves always more additions in the low income groups, with the effect of raising poverty levels for a given poverty line.

Once we consider relative poverty measures, the impact of adding missing persons is, in most cases, indeterminate. Even when the capital dilution effect dominates, it is not necessarily true that adding missing persons raises measured poverty, unlike under absolute poverty measures. The reason is that the addition of poor persons may push the poverty line down, and allow some people to escape from poverty despite the general fall in the incomes.

\section{Some historical comparisons}

The analysis based on our dynamic general equilibrium model suggests that, in theory, income-differentiated mortality has an ambiguous effect on the measurement of poverty. The addition of prematurely dead persons may lead to either a rise or a fall of measures of absolute or relative poverty, depending on whether the capital dilution effect dominates the horizon effect. In the light of this indeterminacy, one may want to take real data, to explore to what extent income-differentiated mortality contributes to reduce measured poverty.

For that purpose, this section proposes a historical illustration based on the case of France (1820-2010). This section calibrates an augmented version of our model (with 10 income classes), so as to fit data on output, population and 
poverty. ${ }^{20}$ Then, once the model is calibrated, we propose to compare actual poverty rates with the hypothetical poverty rates that would have prevailed in the absence of income-differentiated mortality. That comparison will allow us to quantify the extent to which income-differentiated mortality affects the measurement of poverty in a historical perspective.

\subsection{Calibration}

In our 3-period OLG setting, each period lasts 25 years. We will thus consider cohorts born in 1820, 1845, 1870, 1895, 1920, 1945, 1970 and 1995. Since we abstract here from infant mortality, we can interpret $\phi_{i}$ as the probability to survive to age 25 conditionally on having survived to age 5 and $\pi_{i}$ as the probability to survive to age 50 conditionally on having survived to age 25 . Given that survival conditions have strongly varied during the 19th and 20th centuries, our calibration relies on period-specific survival rates, i.e. $\phi_{i t}$ and $\pi_{i t}$.

Note that lifetables of the Human Mortality Database give us only average values for $\phi_{t}=\Sigma n_{i} \phi_{i t}$ and $\pi_{t}=\Sigma n_{i} \pi_{i t}$. In order to calibrate group-specific survival probabilities $\phi_{i t}$ and $\pi_{i t}$, we need to know the structure of heterogeneity, i.e. parameters $n_{i}$. Economic historians underline that there is a substantial concentration of wealth in a few hands in the 19th century. Kesztenbaum and Rosenthal (2012) argue that, on the basis of the real estate census of 1876 in Paris, the rich represented $10 \%$ of households, whereas the poor (who paid little tax or no tax) accounted for $68 \%$ of households. For the sake of generality, we will calibrate the parameters $n_{i}$ by using the $1 / f$ function, a simple version of Zipf's Law, which can be regarded as a discrete version of the Pareto Law. ${ }^{21}$

The next stage consists of calibrating, on the basis of average values $\phi_{t}=$ $\Sigma n_{i} \phi_{i t}$ and $\pi_{t}=\Sigma n_{i} \pi_{i t}$ (taken from the Human Mortality Database) and the weights $n_{i}$, group-specific survival probabilities $\phi_{i t}$ and $\pi_{i t}$. Kesztenbaum and Rosenthal (2012) show that, for France, around year 1880, the average life expectancy at age 5 was equal to 52 years. This was equal to 46 years for the lowest decile (in terms of wealth), and to 57 years for the top decile. Around year 1895, life expectancy at age 5 was equal, on average, to 53.5 years. This was equal to 51 years for the lowest decile, and to 59 for the highest decile. This suggests that the gap between the life expectancies of the rich and the poor is far from constant over time. ${ }^{22}$ Following that empirical evidence, we calibrated group specific survival probabilities $\phi_{i t}$ while allowing for a progressive reduction in the gap between social classes. The calibrated values for $\phi_{i t}$ and $\pi_{i t}$ are provided in the Appendix. The cohort growth factor $g_{t}$ was also calibrated for each

\footnotetext{
${ }^{20}$ For simplicity, we assume here that all income groups save some fraction of their income for the old age, and we also abstract here from public pensions (i.e. we fix $\theta=0$ ).

${ }^{21}$ If the group with the highest occurrence is the very poor, and, then, the second class represent $1 / 2$ of the occurrence of the first (i.e. $n_{2}=\frac{n_{1}}{2}$ ), etc. Hence, normalizing the sum of $n_{i}$ to 1 , we thus have $n_{1}+\frac{n_{1}}{2}+\frac{n_{1}}{3}+\ldots=1$, which yields, in percentages, $n_{1}=0.34$, $n_{2}=0.17$ and $n_{3}=0.11$, etc, up to $n_{10}=0.034$.

22 The income/longevity gradient is less steep nowadays. For instance, Blanpain (2016).shows that white collar men (resp. women) in France live 6 years (resp. 3 years) more than blue collar men (resp. women).
} 
period, so as to fit the population figures in the Human Mortality Database. ${ }^{23}$

Regarding the production process, we calibrate the elasticity of output with respect to capital $\alpha$ to 0.30 , in line with the literature. The TFP parameter $B$ and the skills parameters $\lambda_{i}$ are, in the model, time invariant, but such a constraint makes it difficult to replicate the observed patterns for GDP and poverty rates. Hence we calibrate those parameters for each period. As shown in the Appendix, parameters $B_{t}$ and $\lambda_{i t}$ are set to values allowing for the replication of the pattern of GDP in the Maddison Project (2013), and of the pattern of the headcount poverty rate in Ravallion (2016).

\subsection{Results}

In order to examine how income-differentiated mortality affected poverty measurement, we carry out here some simple historical comparisons. We compare the actual patterns for poverty rates with the hypothetical poverty rates that would have prevailed in the absence of income-differentiated mortality, that is, if all income classes had faced the survival conditions of the top income class.

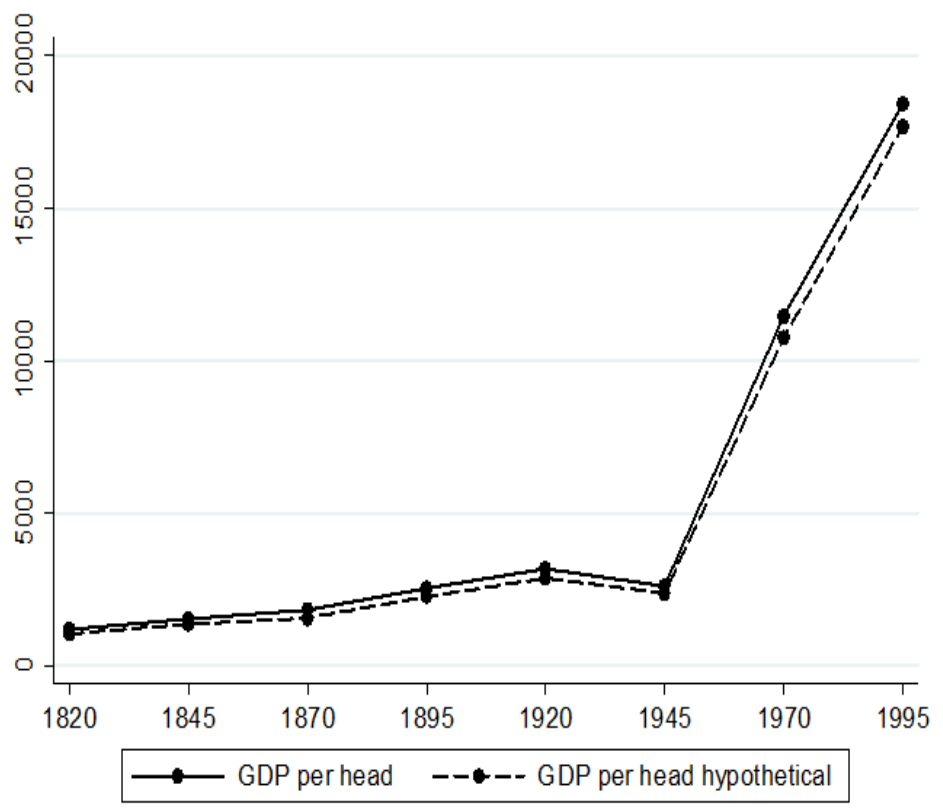

Figure 1: Actual and hypothetical GDP per capita (in 1990 inter GK\$).

When considering that comparison, a central issue underlined in the theoretical model concerns whether the inclusion of the prematurely dead tends to

\footnotetext{
${ }^{23}$ See the Appendix.
} 
increase or decrease income levels. The answer depends on the relative strengths of three distinct effects: the capital dilution effect, the savers partition effect and the horizon effect. As shown on Figure 1, the actual pattern and the hypothetical pattern of GDP per capita are quite close. ${ }^{24}$ Having stressed this, a closer look at the two patterns reveals that the hypothetical GDP patterns tends to lie systematically below the actual one. This implies that the capital dilution effect has, over the period considered, dominated the life horizon effect.

In order to study the impact of income-differentiated mortality on measures of absolute poverty, we will fix an absolute poverty line to its standard level of 1 dollar per day. ${ }^{25}$ Figures 2 and 3 compares, respectively for the young age (i.e. below age 50) and for the old age (i.e. above age 50), the actual headcount poverty rate with the hypothetical headcount poverty rate that would have prevailed without income-differentiated mortality.

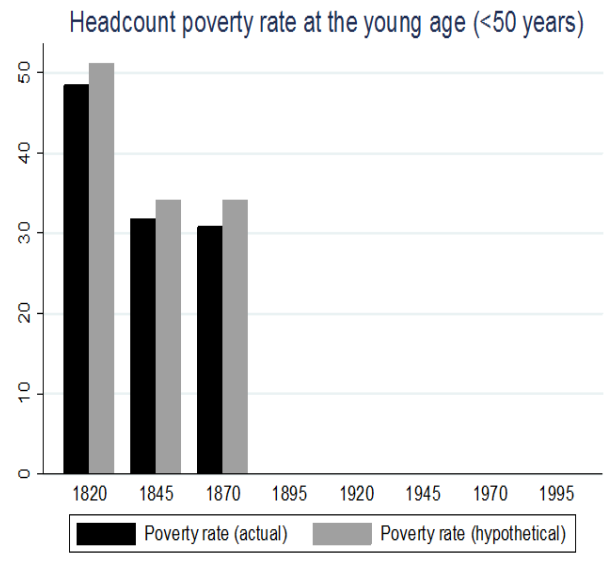

Figure 2: Actual and hypothetical poverty rates at the young age (poverty line $=\$ 1$ a day).

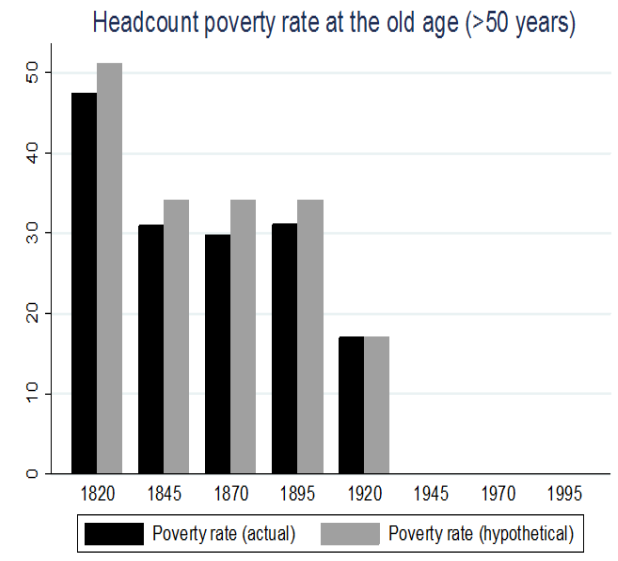

Figure 3: Actual and hypothetical poverty rates at the old age (poverty line $=\$ 1$ a day).

Figures 2 and 3 invite several observations. First, the inclusion of prematurely dead persons tends to increase poverty rates at the young age for 1820 , 1845 and 1870, and at the old age for 1820,1845, 1870 and 1895. Those findings are in line with the theory: when the capital dilution effect dominates the time horizon effect, implying that incomes are pushed down, the inclusion of the prematurely dead raises measures of absolute poverty at the young age and at the old age. Figures 2 and 3 confirm that theoretical result, and add some

\footnotetext{
${ }^{24}$ The reason why the hypothetical GDP pattern is close to the actual one lies in the fact that, under our calibration, the major engine of growth lies in the growth in TFP.

${ }^{25}$ It is assumed here that individuals enjoy the same earnings during the entire periods considered, which are of length 25 years.
} 
quantification of the extent to which adding missing persons increases absolute poverty. The gap between hypothetical and actual poverty rates is equal to about 3 points. This quantifies to what extent income-differentiated mortality tended to bias standard poverty measures downwards.

Another important observation is that the inclusion of prematurely dead persons has a much weaker effect for the measurement of poverty during the 20th century. This result comes partly from the fact that, for those years, the extent of income-differentiated mortality is less substantial, leading to lower adjustments of poverty measures. But there is another explanation, which is due to the level of the poverty line. During the 20th century, the proportion of the French population living with less than 1 dollar per day was low, so that the basis on which the adjustment is made is also quite small. As shown in the Appendix, once the poverty line is fixed to 6 dollars a day (i.e. $\$ 2190$ a year), the gap between actual and hypothetical poverty measures becomes positive also in the first part of the 20th century.

Let us now consider the impact of adding missing persons on the measurement of relative poverty. For that purpose, we follow here the same strategy as above, and compare measures of poverty under the actual survival conditions for each social classes with the hypothetical case where all groups would have enjoyed the same survival conditions as the ones faced by the richest in each cohort. Concerning the choice of a poverty line, we consider here a poverty line fixed to $60 \%$ of the median income within an age group. ${ }^{26}$

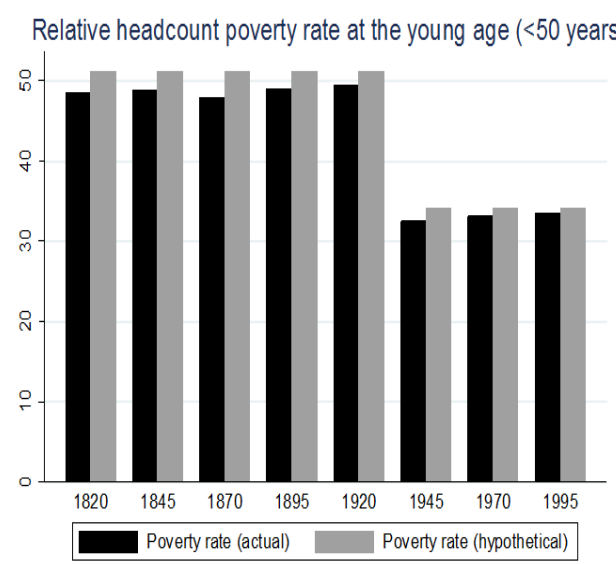

Figure 4: Actual and hypothetical poverty rates at the young age (poverty line $=60 \%$ of median income).

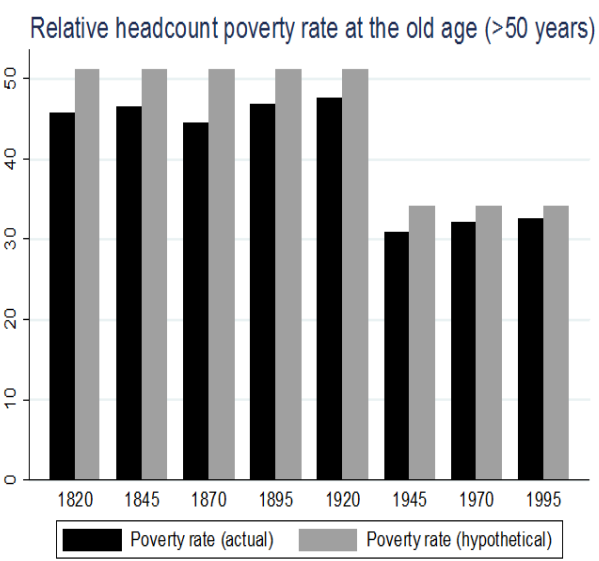

Figure 5: Actual and hypothetical poverty rates at the old age (poverty line $=60 \%$ of median income).

\footnotetext{
${ }^{26}$ In the Appendix, we use a poverty line equal to $40 \%$ of the median income.
} 
As shown on Figure 4, the inclusion of the prematurely dead leads to a rise in measures of relative poverty at the young age. Unlike for absolute poverty measures, that result was not clear in theory. Indeed, when the capital dilution effect dominates, the inclusion of prematurely dead persons has here the additional effect to push the poverty line down, allowing a larger number of persons to escape from poverty. Figure 4 shows that this additional effect is dominated by the rise in the number of persons with lower incomes. When considering measures of relative poverty at the old age (Figure 5), we find that the inclusion of prematurely dead persons leads here to a larger rise in the measured poverty. The gap between the hypothetical and the actual poverty rates above age 50 can, for some years, exceed 5 points.

Those few historical comparisons illustrate that the effects of adding missing persons on poverty measurement may strongly differ when considering measures of absolute or relative poverty. Moreover, our numerical simulations suggest also that the extent to which income-differentiated mortality affects the measurement of poverty varies across periods and age groups.

\section{Concluding remarks}

Income-differentiated mortality raises deep challenges for the measurement of poverty, in the sense that those differentials in survival conditions may lead to selection effects, and, hence, to bias in poverty measures. Following Kanbur and Mukherjee (2007), one remedy consists of adding prematurely dead persons, in such a way as to take the "missing poor" into account.

From a general equilibrium perspective, adding missing persons can have various effects on wages, on capital accumulation and on the distribution of income. Income-differentiated mortality has thus an uncertain impact on measured poverty. The goal of this paper was to examine how income-differentiated mortality affects poverty measures in a dynamic general equilibrium economy.

Our analysis reveals that the inclusion of the prematurely dead has three effects on the income distribution: (1) capital dilution; (2) changing the partition of savers; (3) a horizon effect encouraging savings for a given partition. Those adverse effects imply that, within a dynamic general equilibrium model, the impact of income-differentiated mortality on the measurement of poverty is far from univocal. When the capital dilution effect dominates other effects, income-differentiated mortality reduces measured poverty in absolute terms, but not necessarily in relative terms. When the horizon effect dominates other effects, income-differentiated mortality can either increase or decrease measured poverty, depending on how large the horizon effect is.

Our application to France (1820-2010) shows that, over the last two centuries, the capital dilution effect has dominated other effects, so that the inclusion of prematurely dead persons tends to push incomes down, and to increase measured poverty. Hence, income-differentiated mortality has, over that period, biased standard poverty measures downwards. Moreover, the extent to which standard poverty measures are biased depends on the period, the age group and 
on whether poverty is absolute or relative. The gap between hypothetical and actual poverty rates is larger for the early 19th century, during which standard poverty rates underestimate the prevalence of poverty by about 3 points.

Those findings have an important corollary for the intertemporal comparison of poverty rates. According to our calculations, standard poverty measures, by underestimating the prevalence of poverty in early times, tend to underestimate the extent to which economic development allowed for a reduction of poverty. The selection induced by income-differentiated mortality contributed to make the measured reduction in poverty smaller than the actual one.

In the light of this, it could be tempting to generalize the above claim to any income-based selection process. Note, however, that making such a generalization would be fallacious. Actually, this paper only addressed the issue of income-based selection through differences in mortality, but ignored selection through differences in fertility. In our model, fertility is equal across types. If, on the contrary, we were assuming that (very) poor individuals cannot have children, then it could be possible to have, in theory, an extinction of the (very) poor. ${ }^{27}$ Hence, it could be the case that the observed poverty reduction would not underestimate - as we find here - but overestimate the actual poverty reduction achieved. Hence much work remains to be done, in the future, to quantify to what extent the observed poverty reduction is the corollary of improved standards of living for all, and not the corollary of perverse selection effects.

\section{References}

Ahammer, A., Horvath, T., Winter-Ebmer, R. (2015). The effect of income on mortality: new evidence for the absence of a causal link. IZA Discussion Paper 9176.

Backlund, E., Sorlie, P., Johnson, N. (1999). A comparison of the relationships of education and income with mortality: the national longitudinal mortality study. Social Science and Medicine, 49 (10): 1373-1384.

Belloni, M., Alessie, R., Kalwij, A., Marinacci, C. (2013). Lifetime income and old age mortality risk in Italy over two decades. Demographic Research, 29 (45): 1261-1298.

Blackburn, K., Cipriani, J.P. (2002). A model of longevity, fertility and growth. Journal of Economic Dynamics and Control, 26: 187-204.

Blanplain, N. (2016). L'espérance de vie par catégorie sociale et par diplôme - méthodes et résultats. INSEE, document de travail, F1602.

Boucekkine, R., de la Croix, D., Licandro, O. (2002). Vintage human capital, demographic trends and endogenous growth. Journal of Economic Theory 104:340-375.

Chakraborty, S. (2004) Endogenous lifetime and economic growth. Journal of Economic Theory, 116, 119-137.

de la Croix, D., Licandro, O. (1999). Life expectancy and endogenous growth. Economics Letters 65 (2): 255-263.

\footnotetext{
${ }^{27}$ The intuition is that selection through fertility is cumulative, and could thus be, in theory, much stronger than selection through mortality. In terms of the inclusion of "missing persons", we would have to take into account not only the missing persons, but their missing children, and their missing grandchildren, etc.
} 
de la Croix, D., Licandro, O. (2013). The child is father of the man: Implications for the demographic transition. Economic Journal, 123(567): 236-261.

Deaton, A. (2003) Health, inequality and economic development. Journal of Economic Literature, 41: 113-158.

Deaton, A., Paxson, C. (1998). Aging and Inequality in Income and Health. American Economic Review, 88 (2), 248-253.

Duleep, H.O. (1986) Measuring the effect of income on adult mortality using longitudinal administrative record data. Journal of Human Resources, 21 (2): 238-251.

Evans, W. N., Moore, T. J. (2011). The short-term mortality consequences of income receipt. Journal of Public Economics, 95 (11-12), 1410-1424

Human Mortality Database. University of California, Berkeley (USA), and Max Planck Institute for Demographic Research (Germany). Available at www.mortality.org (data downloaded on January 2016).

Kanbur, R., Mukherjee, D. (2007). Premature mortality and poverty measurement. Bulletin of Economic Research, 59 (4): 339-359.

Kesztenbaum, L., Rosenthal, J.L. (2012). The democratization of longevity: how the poor became old in Paris, 1870-1940, INED.

Lefebvre, M., Pestieau, P., Ponthiere, G. (2013). Measuring Poverty Without the Mortality Paradox. Social Choice and Welfare, 40 (1): 285-316.

Lefebvre, M., Pestieau, P., Ponthiere, G. (2017). FGT old-age poverty measures and the Mortality Paradox: theory and evidence. Review of Income and Wealth, forthcoming.

Maddison Project (2013). http://www.ggdc.net/maddison/maddison-project/home.htm, 2013 version.

Malthus, T. (1798). An Essay on the Principle of Population, London.

Ravallion, M. (2016). The Economics of Poverty. History, Measurement and Policy. Cambridge University Press.

Salm, M. (2007). The effect of pensions on longevity: evidence from Union Army veterans. IZA Discussion Paper 2668.

Sen A.K. (1998). Mortality as an indicator of economic success and failure. Economic Journal 108:1-25

Snyder, S. E., Evans, W. N. (2006). The effect of income on mortality: evidence from the Social Security notch. Review of Economics and Statistics, 88 (3), 482-495.

\section{Appendix}

\subsection{Proof of Proposition 1}

The capital accumulation equation is:

$$
k_{t+1}=\frac{\phi_{2} n_{2} \frac{\pi_{2}}{1+\pi_{2}} \lambda_{2}(1-\alpha)(1-\theta) B k_{t}^{\alpha}+\phi_{3} n_{3} \frac{\pi_{3}}{1+\pi_{3}} \lambda_{3}(1-\alpha)(1-\theta) B k_{t}^{\alpha}}{g A}
$$

This can be rewritten as:

$$
k_{t+1}=\frac{(1-\alpha)(1-\theta) B k_{t}^{\alpha}}{g A}\left(\frac{\phi_{2} \pi_{2} n_{2} \lambda_{2}}{1+\pi_{2}}+\frac{\phi_{3} \pi_{3} n_{3} \lambda_{3}}{1+\pi_{3}}\right) \equiv \varphi\left(k_{t}\right)
$$


The existence of a stationary equilibrium amounts to search for fixed point of $\varphi\left(k_{t}\right)$. We have $\varphi(0)=0$, so that 0 is a stationary equilibrium. Note that we have:

$$
\begin{aligned}
\varphi^{\prime}\left(k_{t}\right) & =\frac{(1-\alpha) \alpha(1-\theta) B k_{t}^{\alpha-1}}{g A}\left(\frac{\phi_{2} \pi_{2} n_{2} \lambda_{2}}{1+\pi_{2}}+\frac{\phi_{3} \pi_{3} n_{3} \lambda_{3}}{1+\pi_{3}}\right)>0 \\
\lim _{k_{t} \rightarrow 0} \varphi^{\prime}\left(k_{t}\right) & =+\infty
\end{aligned}
$$

We thus know that the transition function $\varphi\left(k_{t}\right)$ is above the $45^{\circ}$ line in the $\left(k_{t}, k_{t+1}\right)$ space when $k_{t}$ tends to 0 . We also have:

$$
\lim _{k_{t} \rightarrow+\infty} \frac{\varphi\left(k_{t}\right)}{k_{t}}=\frac{(1-\alpha)(1-\theta) B k_{t}^{\alpha-1}}{g A}\left(\frac{\phi_{2} \pi_{2} n_{2} \lambda_{2}}{1+\pi_{2}}+\frac{\phi_{3} \pi_{3} n_{3} \lambda_{3}}{1+\pi_{3}}\right)=0
$$

Hence, when $k_{t}$ tends to $+\infty$, the $\varphi\left(k_{t}\right)$ function lies below the $45^{\circ}$ line. Hence, given that $\varphi\left(k_{t}\right)$ is above the $45^{\circ}$ line in the $\left(k_{t}, k_{t+1}\right)$ space when $k_{t}$ tends to 0 , and lies below the $45^{\circ}$ line in the $\left(k_{t}, k_{t+1}\right)$ when $k_{t}$ tends to $+\infty$, it must be the case, by continuity, that $\varphi\left(k_{t}\right)$ crosses the $45^{\circ}$ line at least once for some $k_{t}>0$.

Finally, given that

$$
\varphi^{\prime \prime}\left(k_{t}\right)=\frac{(1-\alpha) \alpha(\alpha-1)(1-\theta) B k_{t}^{\alpha-2}}{g A}\left(\frac{\phi_{2} \pi_{2} n_{2} \lambda_{2}}{1+\pi_{2}}+\frac{\phi_{3} \pi_{3} n_{3} \lambda_{3}}{1+\pi_{3}}\right)<0
$$

we know for sure that this intersection is unique. This intersection is achieved when:

$$
k^{*}=\left[\sum_{i=2,3}\left(\frac{\phi_{i} \pi_{i} n_{i} \lambda_{i}}{1+\pi_{i}}\right) \frac{(1-\alpha)(1-\theta) B}{g A}\right]^{\frac{1}{1-\alpha}}
$$

Regarding stability, note that

$$
\varphi^{\prime}(0)=\frac{(1-\alpha) \alpha(1-\theta) B 0^{\alpha-1}}{g A}\left(\frac{\phi_{2} \pi_{2} n_{2} \lambda_{2}}{1+\pi_{2}}+\frac{\phi_{3} \pi_{3} n_{3} \lambda_{3}}{1+\pi_{3}}\right)>1
$$

so that 0 is clearly unstable.

On the contrary, we have

$\varphi^{\prime}\left(k^{*}\right)=\frac{(1-\alpha) \alpha(1-\theta) B\left[\sum_{i=2,3}\left(\frac{\phi_{i} \pi_{i} n_{i} \lambda_{i}}{1+\pi_{i}}\right) \frac{(1-\alpha)(1-\theta) B}{g A}\right]^{\frac{\alpha-1}{1-\alpha}}\left(\sum_{i=2,3} \frac{\phi_{i} n_{i} \pi_{i} \lambda_{i}}{1+\pi_{i}}\right)}{g A}=\alpha<1$

Hence $k^{*}$ is locally stable. 


\subsection{Proof of Proposition 3}

At the stationary equilibrium, we have three income levels at the young age (net of taxes):

$$
\begin{aligned}
& y_{y 1} \equiv \lambda_{1} B(1-\alpha)(1-\theta)\left[\sum_{i=2,3}\left(\frac{\phi_{i} n_{i} \pi_{i} \lambda_{i}}{1+\pi_{i}}\right) \frac{(1-\alpha)(1-\theta) B}{g \Sigma n_{i} \phi_{i} \lambda_{i}}\right]^{\frac{\alpha}{1-\alpha}} \\
& y_{y 2} \equiv \lambda_{2} B(1-\alpha)(1-\theta)\left[\sum_{i=2,3}\left(\frac{\phi_{i} n_{i} \pi_{i} \lambda_{i}}{1+\pi_{i}}\right) \frac{(1-\alpha)(1-\theta) B}{g \Sigma n_{i} \phi_{i} \lambda_{i}}\right]^{\frac{\alpha}{1-\alpha}}=\frac{\lambda_{2}}{\lambda_{1}} y_{y 1} \\
& y_{y 3} \equiv \lambda_{3} B(1-\alpha)(1-\theta)\left[\sum_{i=2,3}\left(\frac{\phi_{i} n_{i} \pi_{i} \lambda_{i}}{1+\pi_{i}}\right) \frac{(1-\alpha)(1-\theta) B}{g \Sigma n_{i} \phi_{i} \lambda_{i}}\right]^{\frac{\alpha}{1-\alpha}}=\frac{\lambda_{3}}{\lambda_{1}} y_{y 1}
\end{aligned}
$$

Hence, we have that the poverty rate $P_{y}$ at the young age can take, in theory, four distinct values, depending on the structural parameters of our economy:

$$
P_{y}=\left\{\begin{array}{l}
0 \text { when } y_{y 1} \geq \omega \\
\frac{\phi_{1} n_{1}}{\sum \phi_{i} n_{i}} \text { when } y_{y 1}<\omega \leq \frac{\lambda_{2}}{\lambda_{1}} y_{y 1} \\
\frac{\phi_{1} n_{1}+\phi_{2} n_{2}}{\Sigma \phi_{i} n_{i}} \text { when } \frac{\lambda_{2}}{\lambda_{1}} y_{y 1}<\omega \leq \frac{\lambda_{3}}{\lambda_{1}} y_{y 1} \\
1 \text { when } \frac{\lambda_{3}}{\lambda_{1}} y_{y 1}<\omega
\end{array}\right.
$$

Thus, for a given poverty line $\omega$, whether a more or less large proportion of young adults lies in poverty depends on technological parameters $(B, \alpha$ and $\left.\lambda_{i}\right)$, on the cohort growth rate $1-g$, on the distribution of skills $\left(n_{i}\right)$ and on survival conditions $\left(\phi_{i}\right.$ and $\left.\pi_{i}\right)$.

Consider now the hypothetical case where all types of individuals benefit from the survival conditions of the richest (i.e. $\phi_{3}$ and $\pi_{3}$ ). In that hypothetical case, the three income levels at the young age are:

$$
\begin{aligned}
\hat{y}_{y 1} & \equiv \lambda_{1} B(1-\alpha)(1-\theta)\left[\sum_{i=2,3}\left(\frac{\phi_{3} n_{i} \pi_{3} \lambda_{i}}{1+\pi_{3}}\right) \frac{(1-\alpha)(1-\theta) B}{g \phi_{3} \Sigma n_{j} \lambda_{j}}\right]^{\frac{\alpha}{1-\alpha}} \\
\hat{y}_{y 2} & \equiv \lambda_{2} B(1-\alpha)(1-\theta)\left[\sum_{i=2,3}\left(\frac{\phi_{3} n_{i} \pi_{3} \lambda_{i}}{1+\pi_{3}}\right) \frac{(1-\alpha)(1-\theta) B}{g \phi_{3} \Sigma n_{j} \lambda_{j}}\right]^{\frac{\alpha}{1-\alpha}}=\frac{\lambda_{2}}{\lambda_{1}} \hat{y}_{y 1} \\
\hat{y}_{y 3} & \equiv \lambda_{3} B(1-\alpha)(1-\theta)\left[\sum_{i=2,3}\left(\frac{\phi_{3} n_{i} \pi_{3} \lambda_{i}}{1+\pi_{3}}\right) \frac{(1-\alpha)(1-\theta) B}{g \phi_{3} \Sigma n_{j} \lambda_{j}}\right]^{\frac{\alpha}{1-\alpha}}=\frac{\lambda_{3}}{\lambda_{1}} \hat{y}_{y 1}
\end{aligned}
$$

The adjusted poverty rate at the young age can take the following values:

$$
\hat{P}_{y}=\left\{\begin{array}{l}
0 \text { when } \hat{y}_{y 1} \geq \omega \\
\frac{n_{1}}{\sum n_{i}} \text { when } \hat{y}_{y 1}<\omega \leq \frac{\lambda_{2}}{\lambda_{1}} \hat{y}_{y 1} \\
\frac{n_{1}+n_{2}}{\Sigma n_{i}} \text { when } \frac{\lambda_{2}}{\lambda_{1}} \hat{y}_{y 1}<\omega \leq \frac{\lambda_{3}}{\lambda_{1}} \hat{y}_{y 1} \\
1 \text { when } \frac{\lambda_{3}}{\lambda_{1}} \hat{y}_{y 1}<\omega
\end{array}\right.
$$


Quite interestingly, the income levels at the young age at the actual stationary equilibrium and at the hypothetical one can be compared as follows. Let us first compare $y_{y 1}$ with $\hat{y}_{y 1}$. We have:

$$
\begin{aligned}
\hat{y}_{y 1}=y_{y 1} \frac{\lambda_{1} B(1-\alpha)(1-\theta)\left[\sum_{i=2,3}\left(\frac{\phi_{3} \pi_{3} n_{i} \lambda_{i}}{1+\pi_{3}}\right) \frac{(1-\alpha)(1-\theta) B}{g \phi_{3} \Sigma n_{j} \lambda_{j}}\right]^{\frac{\alpha}{1-\alpha}}}{\lambda_{1} B(1-\alpha)(1-\theta)\left[\sum_{i=2,3}\left(\frac{\phi_{i} \pi_{i} n_{i} \lambda_{i}}{1+\pi_{i}}\right) \frac{(1-\alpha)(1-\theta) B}{g \Sigma n_{j} \phi_{j} \lambda_{j}}\right]^{\frac{\alpha}{1-\alpha}}} \\
=y_{y 1}\left[\frac{\sum_{i=2,3}\left(\frac{\phi_{3} \pi_{3} n_{i} \lambda_{i}}{1+\pi_{3}}\right) \frac{1}{\sum_{3} \Sigma n_{j} \lambda_{j}}}{\left[\frac{\phi_{i} \pi_{i} n_{i} \lambda_{i}}{1+\pi_{i}}\right) \frac{1}{\Sigma n_{j} \phi_{j} \lambda_{j}}}\right]^{\frac{\alpha}{1-\alpha}}
\end{aligned}
$$

Hence we have: $y_{y 1} \gtrless \hat{y}_{y 1}$ when:

$$
\Delta \equiv \frac{\sum_{i=2,3}\left(\frac{\phi_{3} n_{i} \pi_{3} \lambda_{i}}{1+\pi_{3}}\right) \frac{1}{\sum_{3} \Sigma n_{j} \lambda_{j}}}{\sum_{i=2,3}\left(\frac{\phi_{i} n_{i} \pi_{i} \lambda_{i}}{1+\pi_{i}}\right) \frac{1}{\Sigma n_{j} \phi_{j} \lambda_{j}}} \lessgtr 1
$$

Note that, when $y_{y 1}>\hat{y}_{y 1}$, we have:

$$
\begin{aligned}
& y_{y 2}=\frac{\lambda_{2}}{\lambda_{1}} y_{y 1}>\hat{y}_{y 2}=\frac{\lambda_{2}}{\lambda_{1}} \hat{y}_{y 1}=\frac{\lambda_{2}}{\lambda_{1}} \Delta^{\frac{\alpha}{1-\alpha}} y_{y 1} \\
& y_{y 3}=\frac{\lambda_{3}}{\lambda_{1}} y_{y 1}>\hat{y}_{y 3}=\frac{\lambda_{3}}{\lambda_{1}} \hat{y}_{y 1}=\frac{\lambda_{3}}{\lambda_{1}} \Delta^{\frac{\alpha}{1-\alpha}} y_{y 1}
\end{aligned}
$$

and inverted inequalities when $y_{y 1}<\hat{y}_{y 1}$.

Based on those inequalities, Proposition 3 can be proved by considering the different possible impacts, in terms of poverty measurement, of multiplying the three income levels $y_{y i}$ by $\Delta^{\frac{\alpha}{1-\alpha}}$. We first start by considering all possible cases a priori. The conditions on income levels were first presented in terms of the common component $y_{y 1}$ :

$$
\hat{y}_{y 1}<\omega \Leftrightarrow y_{y 1} \Delta^{\frac{\alpha}{1-\alpha}}<\omega
$$

Then, in a second stage, we deleted the impossible cases, i.e. cases for which conditions on inequalities before the adjustment and after the adjustment contradict themselves. For instance, when considering the case where $y_{y 1}<\omega \leq$ $\frac{\lambda_{2}}{\lambda_{1}} y_{y 1}$, a priori four cases could arise:

- if $y_{y 1} \Delta^{\frac{\alpha}{1-\alpha}} \geq \omega$, then $P_{y}=\frac{\phi_{1} n_{1}}{\Sigma n_{i} \phi_{i}}>\hat{P}_{y}=0$;

- if $y_{y 1} \Delta^{\frac{\alpha}{1-\alpha}}<\omega \leq \frac{\lambda_{2}}{\lambda_{1}} y_{y 1} \Delta^{\frac{\alpha}{1-\alpha}}$, then $P_{y}=\frac{\phi_{1} n_{1}}{\Sigma n_{i} \phi_{i}}<\hat{P}_{y}=\frac{n_{1}}{\Sigma n_{i}}$; 
- if $\frac{\lambda_{2}}{\lambda_{1}} y_{y 1} \Delta^{\frac{\alpha}{1-\alpha}}<\omega \leq \frac{\lambda_{3}}{\lambda_{1}} y_{y 1} \Delta^{\frac{\alpha}{1-\alpha}}$, then $P_{y}=\frac{\phi_{1} n_{1}}{\Sigma n_{i} \phi_{i}}<\hat{P}_{y}=\frac{n_{1}+n_{2}}{\Sigma n_{i}}$;

- if $\frac{\lambda_{3}}{\lambda_{1}} y_{y 1} \Delta^{\frac{\alpha}{1-\alpha}}<\omega$, then $P_{y}=\frac{\phi_{1} n_{1}}{\Sigma n_{i} \phi_{i}}<\hat{P}_{y}=1$;

But clearly, given that $\Delta<1$, it cannot be the case that both $y_{y 1}<\omega$ and $y_{y 1} \Delta^{\frac{\alpha}{1-\alpha}} \geq \omega$, so that impossible case was deleted. Similar cancellations were made for other cases, when there is a conflict between conditions.

Finally, note that, when $\Delta<1$, so that $y_{y i}>\hat{y}_{y i} \forall i=1,2,3$, the extreme cases not treated in Proposition 3 are:

- If $y_{y 1} \geq \omega$

- if $y_{y 1} \Delta^{\frac{\alpha}{1-\alpha}} \geq \omega$, then $P_{y}=\hat{P}_{y}=0$;

- if $y_{y 1} \Delta^{\frac{\alpha}{1-\alpha}}<\omega \leq \frac{\lambda_{2}}{\lambda_{1}} y_{y 1} \Delta^{\frac{\alpha}{1-\alpha}}$, then $P_{y}=0<\hat{P}_{y}=\frac{n_{1}}{\Sigma n_{i}}$;

- if $\frac{\lambda_{2}}{\lambda_{1}} y_{y 1} \Delta^{\frac{\alpha}{1-\alpha}}<\omega \leq \frac{\lambda_{3}}{\lambda_{1}} y_{y 1} \Delta^{\frac{\alpha}{1-\alpha}}$, then $P_{y}=0<\hat{P}_{y}=\frac{n_{1}+n_{2}}{\Sigma n_{i}}$;

- if $\frac{\lambda_{3}}{\lambda_{1}} y_{y 1} \Delta^{\frac{\alpha}{1-\alpha}}<\omega$, then $P_{y}=0<\hat{P}_{y}=1$;

- If $\frac{\lambda_{3}}{\lambda_{1}} y_{y 1}<\omega$, then $P_{y}=\hat{P}_{y}=1$.

\subsection{Proof of Proposition 5}

We have, at the stable stationary equilibrium:

$$
\begin{aligned}
& y_{o 1}=\left[\sum_{i=2,3}\left(\frac{\phi_{i} n_{i} \pi_{i} \lambda_{i}}{1+\pi_{i}}\right) \Omega\right]^{\frac{\alpha}{1-\alpha}} \frac{\theta B(1-\alpha)\left(\Sigma n_{i} \phi_{i} \lambda_{i}\right)}{n_{1} \phi_{1} \pi_{1}} \\
& y_{o i}=\left[\sum_{j=2,3}\left(\frac{\phi_{j} n_{j} \pi_{j}}{1+\pi_{j}} \lambda_{j}\right) \Omega\right]^{\frac{2 \alpha-1}{1-\alpha}}\left(\frac{\lambda_{i}(1-\alpha)(1-\theta) B^{2} \alpha}{1+\pi_{i}}\right) \text { for } i=2,3
\end{aligned}
$$

Hence we have

$$
y_{o 2}=\frac{\lambda_{2}}{1+\pi_{2}} \Xi y_{o 1} \text { and } y_{o 3}=\frac{\lambda_{3}}{1+\pi_{3}} \Xi y_{o 1}
$$

where

$$
\Xi \equiv \frac{g n_{1} \phi_{1} \pi_{1} \alpha}{\theta(1-\alpha)\left(\frac{\phi_{2} n_{2} \pi_{2} \lambda_{2}}{1+\pi_{2}}+\frac{\phi_{3} n_{3} \pi_{3} \lambda_{3}}{1+\pi_{3}}\right)}
$$

Given this, the old-age poverty $P_{o}$ measure takes the following values:

$$
P_{o}=\left\{\begin{array}{l}
0 \text { when } y_{o 1} \geq \omega \\
\frac{\phi_{1} \pi_{1} n_{1}}{\sum \phi_{i} \pi_{i} n_{i}} \text { when } y_{o 1}<\omega \leq \frac{\lambda_{2}}{\left(1+\pi_{2}\right)} \Xi y_{o 1} \\
\frac{\phi_{1} \pi_{1} n_{1}+\phi_{2} \pi_{2} n_{2}}{\Sigma \phi_{i} \pi_{i} n_{i}} \text { when } \frac{\lambda_{2}}{\left(1+\pi_{2}\right)} \Xi y_{o 1}<\omega \leq \frac{\lambda_{3}}{\left(1+\pi_{3}\right)} \Xi y_{o 1} \\
1 \text { when } \frac{\lambda_{3}}{\left(1+\pi_{3}\right)} \Xi y_{o 1}<\omega
\end{array}\right.
$$


Let us now compare those poverty measures with those that would have prevailed in the absence of income differentiated mortality. For that purpose, we now assume that all types of individuals benefit from the survival conditions of the richest (i.e. $\phi_{3}$ and $\pi_{3}$ ). In that hypothetical case, the three income levels at the old age are:

$$
\begin{aligned}
\hat{y}_{o 1} \equiv & {\left[\sum_{i=2,3}\left(\frac{\phi_{3} n_{i} \pi_{3} \lambda_{i}}{1+\pi_{3}}\right) \frac{(1-\alpha)(1-\theta) B}{g \phi_{3} \Sigma n_{j} \lambda_{j}}\right]^{\frac{\alpha}{1-\alpha}} \frac{\theta B(1-\alpha)\left(\Sigma n_{j} \lambda_{j}\right)}{n_{1} \pi_{3}} } \\
\hat{y}_{o 2} \equiv & {\left[\sum_{i=2,3}\left(\frac{\phi_{3} n_{i} \pi_{3} \lambda_{i}}{1+\pi_{3}}\right) \frac{(1-\alpha)(1-\theta) B}{g \phi_{3} \Sigma n_{j} \lambda_{j}}\right]^{\frac{2 \alpha-1}{1-\alpha}} \frac{\lambda_{2}(1-\alpha)(1-\theta) B B \alpha}{1+\pi_{3}} } \\
= & \left.\theta B(1-\alpha)\left(1+\pi_{3}\right)\left[\sum_{i=2,3}\left(\frac{\phi_{3} n_{i} \pi_{3} \lambda_{i}}{1+\pi_{3}}\right)\right]^{\hat{y}_{o 1}}\right]^{2} \\
\hat{y}_{o 3} & \equiv\left[\sum_{i=2,3}\left(\frac{\phi_{3} n_{i} \pi_{3} \lambda_{i}}{1+\pi_{3}}\right) \frac{(1-\alpha)(1-\theta) B}{g \phi_{3} \Sigma n_{j} \lambda_{j}}\right]^{\frac{2 \alpha-1}{1-\alpha}} \frac{\lambda_{3}(1-\alpha)(1-\theta) B B \alpha}{1+\pi_{3}}=\frac{\lambda_{3}}{\lambda_{2}} \hat{y}_{o 2} \\
= & \frac{\lambda_{3}}{\lambda_{2}} \frac{n_{1} \pi_{3} \lambda_{2} B \alpha g \phi_{3}}{\left[\theta B(1-\alpha)\left(1+\pi_{3}\right)\left[\sum_{i=2,3}\left(\frac{\phi_{3} n_{i} \pi_{3} \lambda_{i}}{1+\pi_{3}}\right)\right]\right.} \hat{y}_{o 1}
\end{aligned}
$$

The adjusted old-age poverty $\hat{P}_{o}$ measure takes the following values:

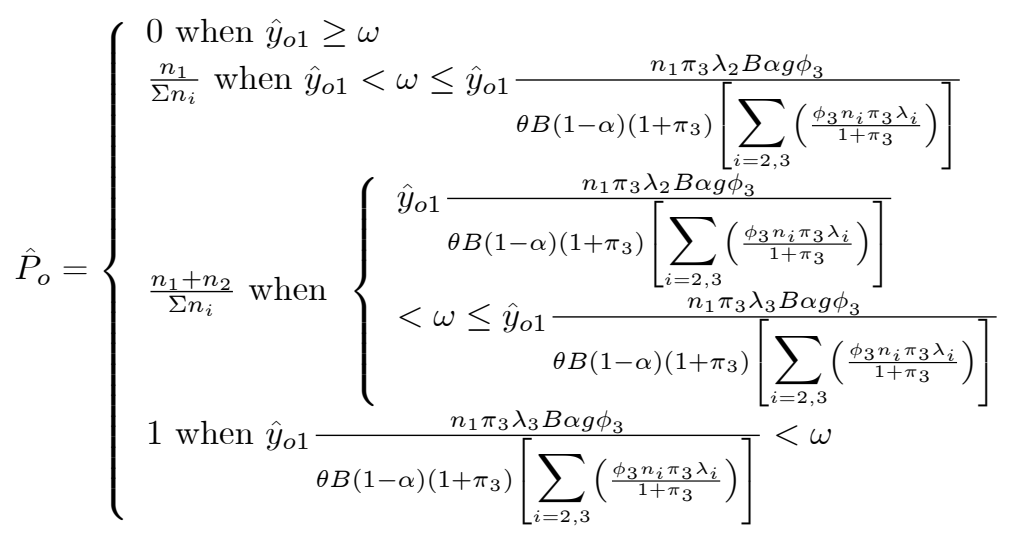


When comparing $y_{o 1}$ with $\hat{y}_{o 1}$, we have:

$$
\begin{aligned}
\hat{y}_{o 1} & \equiv\left[\sum_{i=2,3}\left(\frac{\phi_{3} n_{i} \pi_{3} \lambda_{i}}{1+\pi_{3}}\right) \frac{(1-\alpha)(1-\theta) B}{g \phi_{3} \Sigma n_{j} \lambda_{j}}\right]^{\frac{\alpha}{1-\alpha}} \frac{\theta B(1-\alpha) \phi_{3}\left(\Sigma n_{j} \lambda_{j}\right)}{n_{1} \phi_{3} \pi_{3}} \\
y_{o 1} & \equiv\left[\sum_{i=2,3}\left(\frac{\phi_{i} n_{i} \pi_{i} \lambda_{i}}{1+\pi_{i}}\right) \frac{(1-\alpha)(1-\theta) B}{g \Sigma n_{j} \lambda_{j} \phi_{j}}\right]^{\frac{\alpha}{1-\alpha}} \frac{\theta B(1-\alpha)\left(\Sigma n_{j} \phi_{j} \lambda_{j}\right)}{n_{1} \phi_{1} \pi_{1}}
\end{aligned}
$$

Hence

$$
\hat{y}_{o 1}=y_{o 1} \Delta^{\frac{\alpha}{1-\alpha}} \frac{\phi_{1} \pi_{1}\left(\Sigma n_{j} \lambda_{j}\right)}{\pi_{3}\left(\Sigma n_{i} \phi_{j} \lambda_{j}\right)}
$$

We also have:

$$
\begin{aligned}
& \hat{y}_{o 2}=\left[\sum_{i=2,3}\left(\frac{\phi_{3} n_{i} \pi_{3} \lambda_{i}}{1+\pi_{3}}\right) \frac{(1-\alpha)(1-\theta) B}{g \phi_{3} \Sigma n_{j} \lambda_{j}}\right]^{\frac{2 \alpha-1}{1-\alpha}} \frac{\lambda_{2}\left(\frac{(1-\alpha)(1-\theta) B}{g \phi_{3} \Sigma n_{j} \lambda_{j}}\right) g B \alpha \phi_{3} \Sigma n_{j} \lambda_{j}}{1+\pi_{3}} \\
& y_{o 2}=\left[\sum_{i=2,3}\left(\frac{\phi_{i} n_{i} \pi_{i} \lambda_{i}}{1+\pi_{i}}\right) \frac{(1-\alpha)(1-\theta) B}{g \Sigma n_{j} \lambda_{j} \phi_{j}}\right]^{\frac{2 \alpha-1}{1-\alpha}} \frac{\lambda_{2}\left(\frac{(1-\alpha)(1-\theta) B}{g \Sigma n_{j} \lambda_{j} \phi_{j}}\right) g B \alpha \Sigma n_{j} \phi_{j} \lambda_{j}}{1+\pi_{2}}
\end{aligned}
$$

Hence

$$
\hat{y}_{o 2}=y_{o 2} \Delta^{\frac{2 \alpha-1}{1-\alpha}} \frac{1+\pi_{2}}{1+\pi_{3}}
$$

Hence, given that:

$$
y_{o 2}=\frac{n_{1} \phi_{1} \pi_{1}\left(\lambda_{2} g \Sigma n_{i} \lambda_{i} \phi_{i}\left(\frac{(1-\alpha)(1-\theta) B}{g \Sigma n_{i} \lambda_{i} \phi_{i}}\right) B \alpha\right)}{\theta B(1-\alpha)\left(\Sigma n_{i} \phi_{i} \lambda_{i}\right)\left(1+\pi_{2}\right) \sum_{i=2,3}\left(\frac{\phi_{i} n_{i} \pi_{i} \lambda_{i}}{1+\pi_{i}}\right) \frac{(1-\alpha)(1-\theta) B}{g \Sigma n_{j} \lambda_{j} \phi_{j}}} y_{o 1}
$$

we also have:

$$
\hat{y}_{o 2}=y_{o 1} \Delta^{\frac{2 \alpha-1}{1-\alpha}} \frac{n_{1} \phi_{1} \pi_{1} \lambda_{2} \alpha g}{\theta\left(1+\pi_{3}\right)(1-\alpha) \sum_{i=2,3}\left(\frac{\phi_{i} n_{i} \pi_{i} \lambda_{i}}{1+\pi_{i}}\right)}
$$

We also have:

$$
\begin{aligned}
& y_{o 3}=\left[\sum_{i=2,3}\left(\frac{\phi_{i} n_{i} \pi_{i} \lambda_{i}}{1+\pi_{i}}\right) \frac{(1-\alpha)(1-\theta) B}{g \Sigma n_{j} \lambda_{j} \phi_{j}}\right]^{\frac{2 \alpha-1}{1-\alpha}} \frac{\lambda_{3} g \Sigma n_{j} \lambda_{j} \phi_{j}\left(\frac{(1-\alpha)(1-\theta) B}{g \Sigma n_{j} \lambda_{j} \phi_{j}}\right) B \alpha}{1+\pi_{3}} \\
& \hat{y}_{o 3}=\left[\sum_{i=2,3}\left(\frac{\phi_{3} n_{i} \pi_{3} \lambda_{i}}{1+\pi_{3}}\right) \frac{(1-\alpha)(1-\theta) B}{g \phi_{3} \Sigma n_{j} \lambda_{j}}\right]^{\frac{2 \alpha-1}{1-\alpha}} \frac{\lambda_{3} g \phi_{3} \Sigma n_{j} \lambda_{j}\left(\frac{(1-\alpha)(1-\theta) B}{g \phi_{3} \Sigma n_{j} \lambda_{j}}\right) B \alpha}{1+\pi_{3}}
\end{aligned}
$$


Hence:

$$
\hat{y}_{o 3}=\Delta^{\frac{2 \alpha-1}{1-\alpha}} y_{o 3}
$$

Note also that:

$$
\begin{aligned}
\hat{y}_{o 3} & =\frac{\lambda_{3}}{\lambda_{2}} \hat{y}_{o 2}=\frac{\lambda_{3}}{\lambda_{2}} y_{o 1} \Delta^{\frac{2 \alpha-1}{1-\alpha}} \frac{n_{1} \phi_{1} \pi_{1}\left(\lambda_{2}(((1-\alpha)(1-\theta) B) B \alpha) \frac{\left(1+\pi_{2}\right)}{\left(1+\pi_{3}\right)}\right.}{\theta B(1-\alpha)\left(\Sigma n_{j} \phi_{j} \lambda_{j}\right)\left(1+\pi_{2}\right) \sum_{i=2,3}\left(\frac{\phi_{i} n_{i} \pi_{i} \lambda_{i}}{1+\pi_{i}}\right) \frac{(1-\alpha)(1-\theta) B}{g \Sigma n_{j} \lambda_{j} \phi_{j}}} \\
& =\frac{\lambda_{3}}{\lambda_{2}} y_{o 1} \Delta^{\frac{2 \alpha-1}{1-\alpha}} \frac{n_{1} \phi_{1} \pi_{1} \lambda_{2} \alpha g}{\theta\left(1+\pi_{3}\right)(1-\alpha) \sum_{i=2,3}\left(\frac{\phi_{i} n_{i} \pi_{i} \lambda_{i}}{1+\pi_{i}}\right)}
\end{aligned}
$$

In the following, we denote

$$
\Psi \equiv \frac{n_{1} \phi_{1} \pi_{1} \lambda_{2} \alpha g}{\theta\left(1+\pi_{3}\right)(1-\alpha)\left(\frac{\phi_{2} n_{2} \pi_{2} \lambda_{2}}{1+\pi_{2}}+\frac{\phi_{3} n_{3} \pi_{3} \lambda_{3}}{1+\pi_{3}}\right)}
$$

, to have

$$
\begin{aligned}
& \hat{y}_{o 1}=y_{o 1} \Delta^{\frac{\alpha}{1-\alpha}} \frac{\phi_{1} \pi_{1}\left(\sum n_{i} \lambda_{i}\right)}{\pi_{3}\left(\sum n_{i} \phi_{i} \lambda_{i}\right)} \\
& \hat{y}_{o 2}=y_{o 1} \Delta^{\frac{2 \alpha-1}{1-\alpha}} \Psi \\
& \hat{y}_{o 3}=\frac{\lambda_{3}}{\lambda_{2}} y_{o 1} \Delta^{\frac{2 \alpha-1}{1-\alpha}} \Psi
\end{aligned}
$$

where

$$
\Psi \equiv \frac{n_{1} \phi_{1} \pi_{1} \lambda_{2} \alpha g}{\theta\left(1+\pi_{3}\right)(1-\alpha)\left(\frac{\phi_{2} n_{2} \pi_{2} \lambda_{2}}{1+\pi_{2}}+\frac{\phi_{3} n_{3} \pi_{3} \lambda_{3}}{1+\pi_{3}}\right)}
$$

Based on those rewritings, we can now complete the proof of Proposition 5, by considering the different possible impacts, in terms of poverty measurement, of multiplying the three income levels at the old age by the corresponding adjustment factors. For that purpose, we suppose, without loss of generality, that $\hat{y}_{o 1}<\hat{y}_{o 2}, \hat{y}_{o 3}$. We first start by considering all possible cases a priori. The conditions on income levels were first presented in common terms as:

$$
\hat{y}_{o 1}<\omega \Longleftrightarrow y_{o 1} \Delta^{\frac{\alpha}{1-\alpha}} \frac{\phi_{1} \pi_{1}\left(\Sigma n_{i} \lambda_{i}\right)}{\pi_{3}\left(\Sigma n_{i} \phi_{i} \lambda_{i}\right)}<\omega
$$

We also deleted cases for which the conditions cannot be satisfied. For instance, in the case $\frac{\lambda_{2}}{1+\pi_{2}} \Xi y_{o 1}<\omega \leq \frac{\lambda_{3}}{1+\pi_{3}} \Xi y_{o 1}$, a priori four cases could arise:

- if $y_{o 1} \Delta^{\frac{\alpha}{1-\alpha}} \frac{\phi_{1} \pi_{1}\left(\Sigma n_{i} \lambda_{i}\right)}{\pi_{3}\left(\Sigma n_{i} \phi_{i} \lambda_{i}\right)} \geq \omega$, then $P_{o}=\frac{\phi_{1} \pi_{1} n_{1}+\phi_{2} \pi_{2} n_{2}}{\Sigma \pi_{i} \phi_{i} n_{i}}>\hat{P}_{o}=0$; 
- if $y_{o 1} \Delta^{\frac{\alpha}{1-\alpha}} \frac{\phi_{1} \pi_{1}\left(\Sigma n_{i} \lambda_{i}\right)}{\pi_{3}\left(\Sigma n_{i} \phi_{i} \lambda_{i}\right)}<\omega \leq y_{o 1} \Delta^{\frac{2 \alpha-1}{1-\alpha}} \Psi$, then $P_{o}=\frac{\phi_{1} \pi_{1} n_{1}+\phi_{2} \pi_{2} n_{2}}{\Sigma \pi_{i} \phi_{i} n_{i}} \lessgtr$ $\hat{P}_{o}=\frac{n_{1}}{\Sigma n_{i}}$

- if $y_{o 1} \Delta^{\frac{2 \alpha-1}{1-\alpha}} \Psi<\omega \leq \frac{\lambda_{3}}{\lambda_{2}} y_{o 1} \Delta^{\frac{2 \alpha-1}{1-\alpha}} \Psi$, then $P_{o}=\frac{\phi_{1} \pi_{1} n_{1}+\phi_{2} \pi_{2} n_{2}}{\Sigma \pi_{i} \phi_{i} n_{i}}<\hat{P}_{o}=$ $\frac{n_{1}+n_{2}}{\Sigma n_{i}}$;

- if $\frac{\lambda_{3}}{\lambda_{2}} y_{o 1} \Delta^{\frac{2 \alpha-1}{1-\alpha}} \Psi<\omega$, then $P_{o}=\frac{\phi_{1} \pi_{1} n_{1}+\phi_{2} \pi_{2} n_{2}}{\Sigma \pi_{i} \phi_{i} n_{i}}<\hat{P}_{o}=1$;

But it is clear that, given $\frac{\lambda_{2}}{1+\pi_{2}} \Xi y_{o 1}<\omega \leq \frac{\lambda_{3}}{1+\pi_{3}} \Xi y_{o 1}$, the first two cases cannot arise. These were thus deleted. Similar deletions were made for other logically impossible cases.

The extreme cases not treated in Proposition 5 are:

- If $y_{o 1} \geq \omega$

- if $y_{o 1} \Delta^{\frac{\alpha}{1-\alpha}} \frac{\phi_{1} \pi_{1}\left(\Sigma n_{i} \lambda_{i}\right)}{\pi_{3}\left(\Sigma n_{i} \phi_{i} \lambda_{i}\right)} \geq \omega$, then $P_{o}=\hat{P}_{o}=0$;

- if $y_{o 1} \Delta^{\frac{\alpha}{1-\alpha}} \frac{\phi_{1} \pi_{1}\left(\sum n_{i} \lambda_{i}\right)}{\pi_{3}\left(\Sigma n_{i} \phi_{i} \lambda_{i}\right)}<\omega \leq y_{o 1} \Delta^{\frac{2 \alpha-1}{1-\alpha}} \Psi$, then $P_{o}=0<\hat{P}_{o}=\frac{n_{1}}{\Sigma n_{i}}$;

- if $y_{o 1} \Delta^{\frac{2 \alpha-1}{1-\alpha}} \Psi<\omega \leq \frac{\lambda_{3}}{\lambda_{2}} y_{o 1} \Delta^{\frac{2 \alpha-1}{1-\alpha}} \Psi$, then $P_{o}=0<\hat{P}_{o}=\frac{n_{1}+n_{2}}{\Sigma n_{i}}$;

- if $\frac{\lambda_{3}}{\lambda_{2}} y_{o 1} \Delta^{\frac{2 \alpha-1}{1-\alpha}} \Psi<\omega$, then $P_{o}=0<\hat{P}_{o}=1$;

- If $\frac{\pi_{3} \lambda_{3}}{1+\pi_{3}} \Xi y_{o 1}<\omega$, then $P_{o}=\hat{P}_{o}=1$.

\subsection{Proof of Proposition 7}

The relative poverty rate can take various levels, depending on the level of the median income $y_{y m}$ :

$$
p_{y}=\left\{\begin{array}{l}
0 \text { if } y_{y m}=y_{y 1} \\
0 \text { if } y_{y m}=y_{y 2} \text { and } y_{y 1} \geq x y_{y 2} \\
\frac{\phi_{1} n_{1}}{\Sigma \phi_{i} n_{i}} \text { if } y_{y m}=y_{y 2} \text { and } y_{y 1}<x y_{y 2} \\
0 \text { if } y_{y m}=y_{y 3} \text { and } y_{y 1} \geq x y_{y 3} \\
\frac{\phi_{1} n_{1}}{\Sigma \phi_{i} n_{i}} \text { if } y_{y m}=y_{y 3} \text { and } y_{y 1}<x y_{y 3} \leq y_{y 2} \\
\frac{\phi_{1} n_{1}+\phi_{2} n_{2}}{\Sigma \phi_{i} n_{i}} \text { if } y_{y m}=y_{y 3} \text { and } y_{y 1}<y_{y 2} \leq x y_{y 3}
\end{array}\right.
$$

When considering the economy after the adjustment, we have also several possible cases, depending on where the median (adjusted) income $\hat{y}_{y m}$ lies:

$$
\hat{p}_{y}=\left\{\begin{array}{l}
0 \text { if } \hat{y}_{y m}=\hat{y}_{y 1} \\
0 \text { if } \hat{y}_{y m}=\hat{y}_{y 2} \text { and } \hat{y}_{y 1} \geq x \hat{y}_{y 2} \\
\frac{n_{1}}{\Sigma n_{i}} \text { if } \hat{y}_{y m}=\hat{y}_{y 2} \text { and } \hat{y}_{y 1}<x \hat{y}_{y 2} \\
0 \text { if } \hat{y}_{y m}=\hat{y}_{y 3} \text { and } \hat{y}_{y 1} \geq x \hat{y}_{y 3} \\
\frac{n_{1}}{\sum n_{i}} \text { if } \hat{y}_{y m}=\hat{y}_{y 3} \text { and } \hat{y}_{y 1}<x \hat{y}_{y 3} \leq \hat{y}_{y 2} \\
\frac{n_{1}+n_{2}}{\Sigma n_{i}} \text { if } \hat{y}_{y m}=\hat{y}_{y 3} \text { and } \hat{y}_{y 1}<\hat{y}_{y 2} \leq x \hat{y}_{y 3}
\end{array}\right.
$$


The comparison of $p_{y}$ and $\hat{p}_{y}$ can be carried out by reminding that the income levels of the three classes are related as follows:

$$
\begin{aligned}
& y_{y 2}=\frac{\lambda_{2}}{\lambda_{1}} y_{y 1} ; y_{y 3}=\frac{\lambda_{3}}{\lambda_{1}} y_{y 1} \\
& \hat{y}_{y 1}=y_{y 1} \Delta^{\frac{\alpha}{1-\alpha}} ; \hat{y}_{y 2}=\frac{\lambda_{2}}{\lambda_{1}} y_{y 1} \Delta^{\frac{\alpha}{1-\alpha}} ; \hat{y}_{y 3}=\frac{\lambda_{3}}{\lambda_{1}} y_{y 1} \Delta^{\frac{\alpha}{1-\alpha}}
\end{aligned}
$$

The proof of Proposition 7 follows from merely considering the different cases regarding the initial poverty line and initial extent of poverty, and regarding the new poverty line and the impact of adding missing persons on the income levels. We first start by considering all possible cases a priori. The conditions on income levels were first presented in common terms as:

$$
\hat{y}_{y 1}<x \hat{y}_{y 2} \Longleftrightarrow y_{y 1} \Delta^{\frac{\alpha}{1-\alpha}}<x \frac{\lambda_{2}}{\lambda_{1}} y_{y 1} \Delta^{\frac{\alpha}{1-\alpha}} \Longleftrightarrow 1<x \frac{\lambda_{2}}{\lambda_{1}}
$$

Then, in a second stage, we deleted the impossible cases, i.e. cases for which conditions on inequalities before the adjustment and after the adjustment contradict themselves. For instance, when considering the case where $y_{y m}=y_{y 2}$ and $1<x \frac{\lambda_{2}}{\lambda_{1}}$, a priori three cases could arise:

- if $\hat{y}_{y m}=\hat{y}_{y 1}$, then $p_{y}=\hat{p}_{y}=0$;

- if $\hat{y}_{y m}=\hat{y}_{y 2}$ and $1 \geq x \frac{\lambda_{2}}{\lambda_{1}}$, then $p_{y}=\hat{p}_{y}=0$;

- if $\hat{y}_{y m}=\hat{y}_{y 2}$ and $1<x \frac{\lambda_{2}}{\lambda_{1}}$, then $p_{y}<\hat{p}_{y}=\frac{n_{1}}{\Sigma n_{i}}$;

But the second case cannot arise, since we postulated that $1<x \frac{\lambda_{2}}{\lambda_{1}}$. Hence that case was deleted. Similar deletion were made for other cases.

Finally, note that the extreme cases not treated in Proposition 7 are:

- If $y_{y m}=y_{y 1}$, then $\hat{y}_{y m}=\hat{y}_{y 1}$, so that $p_{y}=\hat{p}_{y}=0$;

- If $y_{y m}=y_{y 2}$ and $1 \geq x \frac{\lambda_{2}}{\lambda_{1}}$,

- if $\hat{y}_{y m}=\hat{y}_{y 1}$, then $p_{y}=\hat{p}_{y}=0$;

- if $\hat{y}_{y m}=\hat{y}_{y 2}$ and given $1 \geq x \frac{\lambda_{2}}{\lambda_{1}}$, then $p_{y}=\hat{p}_{y}=0$;

- If $y_{y m}=y_{y 3}$ and $1 \geq x \frac{\lambda_{3}}{\lambda_{1}}$,

- if $\hat{y}_{y m}=\hat{y}_{y 1}$, then $p_{y}=\hat{p}_{y}=0$;

- if $\hat{y}_{y m}=\hat{y}_{y 2}$ and given $1 \geq x \frac{\lambda_{2}}{\lambda_{1}}$, then $p_{y}=\hat{p}_{y}=0$;

- if $\hat{y}_{y m}=\hat{y}_{y 3}$ and $1 \geq x \frac{\lambda_{3}}{\lambda_{1}}$, then $p_{y}=\hat{p}_{y}=0$. 


\subsection{Proof of Proposition 9}

The relative poverty rate at the old age before the adjustment can take the following values:

$$
p_{o}=\left\{\begin{array}{l}
0 \text { if } y_{o m}=y_{o 1} \\
0 \text { if } y_{o m}=y_{o 2} \text { and } y_{o 1} \geq x y_{o 2} \\
\frac{\pi_{1} \phi_{1} n_{1}}{\Sigma \phi_{i} \pi_{i} n_{i}} \text { if } y_{o m}=y_{o 2} \text { and } y_{o 1}<x y_{o 2} \\
0 \text { if } y_{o m}=y_{o 3} \text { and } y_{o 1} \geq x y_{o 3} \\
\frac{\pi_{1} \phi_{1} n_{1}}{\sum \phi_{i} \pi_{i} n_{i}} \text { if } y_{o m}=y_{o 3} \text { and } y_{o 1}<x y_{o 3} \leq y_{o 2} \\
\frac{\pi_{1} \phi_{1} n_{1}+\pi_{2} \phi_{2} n_{2}}{\Sigma \phi_{i} \pi_{i} n_{i}} \text { if } y_{o m}=y_{y 3} \text { and } y_{o 1}<y_{o 2} \leq x y_{o 3}
\end{array}\right.
$$

When considering the economy after the adjustment, we have also several possible cases, depending on where the median (adjusted) income $\hat{y}_{\text {om }}$ lies:

$$
\hat{p}_{o}=\left\{\begin{array}{l}
0 \text { if } \hat{y}_{y o}=\hat{y}_{o 1} \\
0 \text { if } \hat{y}_{o m}=\hat{y}_{o 2} \text { and } \hat{y}_{o 1} \geq x \hat{y}_{o 2} \\
\frac{n_{1}}{\Sigma n_{i}} \text { if } \hat{y}_{o m}=\hat{y}_{o 2} \text { and } \hat{y}_{o 1}<x \hat{y}_{o 2} \\
0 \text { if } \hat{y}_{o m}=\hat{y}_{o 3} \text { and } \hat{y}_{o 1} \geq x \hat{y}_{o 3} \\
\frac{n_{1}}{\sum n_{i}} \text { if } \hat{y}_{o m}=\hat{y}_{o 3} \text { and } \hat{y}_{o 1}<x \hat{y}_{o 3} \leq \hat{y}_{o 2} \\
\frac{n_{1}+n_{2}}{\Sigma n_{i}} \text { if } \hat{y}_{o m}=\hat{y}_{o 3} \text { and } \hat{y}_{o 1}<\hat{y}_{o 2} \leq x \hat{y}_{o 3}
\end{array}\right.
$$

The comparison of $p_{o}$ and $\hat{p}_{o}$ can be carried out by first reminding that:

$$
\begin{aligned}
y_{o 2} & =\frac{\lambda_{2}}{1+\pi_{2}} \Xi y_{o 1} ; y_{o 3}=\frac{\lambda_{3}}{1+\pi_{3}} \Xi y_{o 1} \\
\hat{y}_{o 1} & =y_{o 1} \Delta^{\frac{\alpha}{1-\alpha}} \frac{\phi_{1} \pi_{1}\left(\Sigma n_{i} \lambda_{i}\right)}{\pi_{3}\left(\Sigma n_{i} \phi_{i} \lambda_{i}\right)} \\
\hat{y}_{o 2} & =y_{o 1} \Delta^{\frac{2 \alpha-1}{1-\alpha}} \Psi \\
\hat{y}_{o 3} & =\frac{\lambda_{3}}{\lambda_{2}} y_{o 1} \Delta^{\frac{2 \alpha-1}{1-\alpha}} \Psi
\end{aligned}
$$

The proof of Proposition 9 follows from merely considering the different cases regarding the initial poverty line and initial extent of poverty, and regarding the new poverty line and the impact of adding missing persons on the income levels. We first start by considering all possible cases a priori. The conditions on income levels were first presented as:

$$
\hat{y}_{o 1} \geq x \hat{y}_{o 2} \Longleftrightarrow \Delta^{\frac{\alpha}{1-\alpha}} \frac{\phi_{1} \pi_{1}\left(\Sigma n_{i} \lambda_{i}\right)}{\pi_{3}\left(\Sigma n_{i} \phi_{i} \lambda_{i}\right)} \geq x \Delta^{\frac{2 \alpha-1}{1-\alpha}} \Psi
$$


Further simplifications were made by replacing for $\Delta$ and $\Psi$, to obtain:

$$
\begin{aligned}
\frac{\phi_{1} \pi_{1}\left(\Sigma n_{j} \lambda_{j}\right)}{\pi_{3}\left(\Sigma n_{j} \phi_{j} \lambda_{j}\right)} & \geq x \frac{n_{1} \phi_{1} \pi_{1} \lambda_{2}(1-\theta) B \alpha\left(\frac{\sum_{i=2,3}\left(\frac{\phi_{i} n_{i} \pi_{i} \lambda_{i}}{1+\pi_{i}}\right) \frac{1}{\Sigma n_{j} \phi_{j} \lambda_{j}}}{\sum_{i=2,3}\left(\frac{\phi_{3} n_{i} \pi_{3} \lambda_{i}}{1+\pi_{3}}\right) \frac{1}{\phi_{3} \Sigma n_{j} \lambda_{j}}}\right)}{\theta\left(1+\pi_{3}\right)\left(\Sigma n_{j} \phi_{j} \lambda_{j}\right) \sum_{i=2,3}\left(\frac{\phi_{i} n_{i} \pi_{i} \lambda_{i}}{1+\pi_{i}}\right) \frac{(1-\alpha)(1-\theta) B}{g \Sigma n_{j} \lambda_{j} \phi_{j}}} \\
\frac{\theta(1-\alpha)}{g} & \geq \frac{x n_{1} \lambda_{2} \alpha}{n_{2} \lambda_{2}+n_{3} \lambda_{3}}
\end{aligned}
$$

We then checked to see whether there was any contradiction between the different conditions, so as to delete logically impossible cases.

Finally, note that the extreme cases not treated in Proposition 9 are:

- If $y_{o m}=y_{o 1}$, then $\hat{y}_{o m}=\hat{y}_{o 1}$, so that $p_{o}=\hat{p}_{o}=0$;

- If $y_{o m}=y_{o 2}$ and $1 \geq x \frac{\pi_{2} \lambda_{2}}{\left(1+\pi_{2}\right)} \Xi$,

- if $\hat{y}_{o m}=\hat{y}_{o 1}$, then $p_{o}=\hat{p}_{o}=0$

- if $\hat{y}_{o m}=\hat{y}_{o 2}$ and $\frac{\theta(1-\alpha)}{\pi_{3} g} \geq \frac{x n_{1} \lambda_{2} \alpha}{\left(n_{2} \lambda_{2}+n_{3} \lambda_{3}\right)}$, then $p_{o}=\hat{p}_{o}=0$;

- if $\hat{y}_{o m}=\hat{y}_{o 2}$ and $\frac{\theta(1-\alpha)}{\pi_{3} g}<\frac{x n_{1} \lambda_{2} \alpha}{\left(n_{2} \lambda_{2}+n_{3} \lambda_{3}\right)} \leq \frac{n_{1} \lambda_{3} \alpha}{\left(n_{2} \lambda_{2}+n_{3} \lambda_{3}\right)}$, then $p_{o}=0<$ $\hat{p}_{o}=\frac{n_{1}}{\Sigma n_{i}}$;

- If $y_{o m}=y_{o 3}$ and $1 \geq x \frac{\pi_{3} \lambda_{3}}{\left(1+\pi_{3}\right)} \Xi$,

- if $\hat{y}_{o m}=\hat{y}_{o 1}$, then $p_{o}=\hat{p}_{o}=0$

- if $\hat{y}_{o m}=\hat{y}_{o 2}$ and if $\frac{\theta(1-\alpha)}{\pi_{3} g} \geq \frac{x n_{1} \lambda_{2} \alpha}{\left(n_{2} \lambda_{2}+n_{3} \lambda_{3}\right)}$, then $p_{o}=\hat{p}_{o}=0$;

- if $\hat{y}_{o m}=\hat{y}_{o 2}$ and if $\frac{\theta(1-\alpha)}{\pi_{3} g}<\frac{x n_{1} \lambda_{2} \alpha}{\left(n_{2} \lambda_{2}+n_{3} \lambda_{3}\right)} \leq \frac{n_{1} \lambda_{3} \alpha}{\left(n_{2} \lambda_{2}+n_{3} \lambda_{3}\right)}$, then $p_{o}=$ $0<\hat{p}_{o}=\frac{n_{1}}{\Sigma n_{i}}$

- if $\hat{y}_{o m}=\hat{y}_{o 3}$ and $\frac{\theta(1-\alpha)}{\pi_{3} g} \geq \frac{x n_{1} \lambda_{3} \alpha}{\left(n_{2} \lambda_{2}+n_{3} \lambda_{3}\right)}$, then $p_{o}=\hat{p}_{o}=0$;

- if $\hat{y}_{o m}=\hat{y}_{o 3}$ and $\frac{\theta(1-\alpha)}{\pi_{3} g}<\frac{x n_{1} \lambda_{3} \alpha}{\left(n_{2} \lambda_{2}+n_{3} \lambda_{3}\right)} \leq \frac{n_{1} \lambda_{2} \alpha}{\left(n_{2} \lambda_{2}+n_{3} \lambda_{3}\right)}$, then $p_{o}=0<$ $\hat{p}_{o}=\frac{n_{1}}{\Sigma n_{i}}$;

- if $\hat{y}_{o m}=\hat{y}_{o 3}$ and $\frac{\theta(1-\alpha)}{\pi_{3} g}<\frac{n_{1} \lambda_{2} \alpha}{\left(n_{2} \lambda_{2}+n_{3} \lambda_{3}\right)}<\frac{x n_{1} \lambda_{3} \alpha}{\left(n_{2} \lambda_{2}+n_{3} \lambda_{3}\right)}$, then $p_{o}=0<$ $\hat{p}_{o}=\frac{n_{1}+n_{2}}{\Sigma n_{i}}$. 


\subsection{Calibration}

\begin{tabular}{lllllllllll}
\hline \hline Cohorts & $\phi_{1 t}$ & $\phi_{2 t}$ & $\phi_{3 t}$ & $\phi_{4 t}$ & $\phi_{5 t}$ & $\phi_{6 t}$ & $\phi_{7 t}$ & $\phi_{8 t}$ & $\phi_{9 t}$ & $\phi_{10 t}$ \\
1820 & 0,552 & 0,599 & 0,605 & 0,611 & 0,612 & 0,630 & 0,643 & 0,674 & 0,688 & 0,707 \\
1845 & 0,599 & 0,643 & 0,648 & 0,654 & 0,656 & 0,672 & 0,684 & 0,712 & 0,726 & 0,743 \\
1870 & 0,496 & 0,548 & 0,555 & 0,562 & 0,564 & 0,583 & 0,598 & 0,631 & 0,648 & 0,668 \\
1895 & 0,642 & 0,685 & 0,691 & 0,696 & 0,698 & 0,714 & 0,727 & 0,755 & 0,769 & 0,777 \\
1920 & 0,705 & 0,743 & 0,748 & 0,752 & 0,754 & 0,768 & 0,779 & 0,804 & 0,817 & 0,832 \\
1945 & 0,741 & 0,777 & 0,782 & 0,786 & 0,788 & 0,802 & 0,812 & 0,836 & 0,848 & 0,863 \\
1970 & 0,899 & 0,927 & 0,930 & 0,934 & 0,935 & 0,946 & 0,954 & 0,972 & 0,981 & 0,993 \\
1995 & 0,967 & 0,992 & 0,995 & 0,998 & 0,999 & 1,000 & 1,000 & 1,000 & 1,000 & 1,000 \\
\hline \hline Cohorts & $\pi_{1 t}$ & $\pi_{2 t}$ & $\pi_{3 t}$ & $\pi_{4 t}$ & $\pi_{5 t}$ & $\pi_{6 t}$ & $\pi_{7 t}$ & $\pi_{8 t}$ & $\pi_{9 t}$ & $\pi_{10 t}$ \\
1820 & 0,493 & 0,535 & 0,540 & 0,545 & 0,547 & 0,563 & 0,575 & 0,602 & 0,615 & 0,631 \\
1845 & 0,538 & 0,577 & 0,582 & 0,587 & 0,588 & 0,603 & 0,614 & 0,639 & 0,652 & 0,667 \\
1870 & 0,427 & 0,472 & 0,478 & 0,483 & 0,485 & 0,502 & 0,515 & 0,543 & 0,557 & 0,574 \\
1895 & 0,556 & 0,594 & 0,598 & 0,603 & 0,605 & 0,619 & 0,630 & 0,654 & 0,666 & 0,673 \\
1920 & 0,629 & 0,663 & 0,667 & 0,671 & 0,673 & 0,685 & 0,695 & 0,718 & 0,728 & 0,742 \\
1945 & 0,655 & 0,687 & 0,691 & 0,695 & 0,697 & 0,709 & 0,718 & 0,739 & 0,750 & 0,763 \\
1970 & 0,855 & 0,881 & 0,885 & 0,888 & 0,889 & 0,899 & 0,907 & 0,924 & 0,933 & 0,944 \\
1995 & 0,928 & 0,951 & 0,954 & 0,957 & 0,958 & 0,967 & 0,974 & 0,990 & 0,998 & 1,000 \\
\hline \hline
\end{tabular}

Table A1 : Calibration of survival probabilities

In order to fit the data for population size from the Human Mortality database, the parameter $g_{t}$ is calibrated as follows.

\begin{tabular}{ccccccccc}
\hline \hline cohorts & 1820 & 1845 & 1870 & 1895 & 1920 & 1945 & 1970 & 1995 \\
population size (x1000) & 3081 & 3508 & 3841 & 3844 & 3253 & 3928 & 4642 & 5568 \\
$g_{t}$ & 1,09 & 1,31 & 1,16 & 0,93 & 1,09 & 1,12 & 1,3 & - \\
\hline \hline
\end{tabular}

Table A2: Calibration of cohort growth rate.

The skills parameters $\lambda_{i t}$ are calibrated in such a way as to be as close as possible to the headcount poverty rates in Ravallion (2016) over 1820-1995.

\begin{tabular}{lllllllllll}
\hline \hline Cohorts & $\lambda_{1 t}$ & $\lambda_{2 t}$ & $\lambda_{3 t}$ & $\lambda_{4 t}$ & $\lambda_{5 t}$ & $\lambda_{6 t}$ & $\lambda_{7 t}$ & $\lambda_{8 t}$ & $\lambda_{9 t}$ & $\lambda_{10 t}$ \\
1820 & 0,10 & 0,10 & 0,50 & 0,70 & 1,00 & 1,10 & 1,30 & 1,60 & 1,90 & 2,50 \\
1845 & 0,10 & 0,30 & 0,50 & 0,70 & 1,00 & 1,10 & 1,30 & 1,60 & 1,90 & 2,50 \\
1870 & 0,10 & 0,30 & 0,50 & 0,70 & 1,00 & 1,10 & 1,30 & 1,60 & 1,90 & 2,50 \\
1895 & 0,10 & 0,30 & 0,50 & 0,70 & 1,00 & 1,10 & 1,30 & 1,60 & 1,90 & 2,50 \\
1920 & 0,20 & 0,30 & 0,50 & 0,70 & 1,00 & 1,10 & 1,30 & 1,60 & 1,90 & 2,50 \\
1945 & 0,20 & 0,50 & 0,50 & 0,70 & 1,00 & 1,10 & 1,30 & 1,60 & 1,90 & 3,50 \\
1970 & 0,40 & 0,70 & 0,80 & 0,90 & 1,00 & 1,10 & 1,60 & 2,00 & 2,50 & 3,50 \\
1995 & 0,40 & 0,70 & 0,80 & 0,90 & 1,00 & 1,10 & 1,60 & 2,00 & 2,50 & 3,50 \\
\hline \hline
\end{tabular}


Starting from an initial $k_{1820}$ fixed to 100 , we can, given the calibrated values for parameters $n_{i}, \lambda_{i t}, \phi_{i t}, \pi_{i t}, \alpha$, and $g_{t}$, find a level of the TFP parameter $B_{t}$ for each period that allows us to perfectly fit the GDP per capita pattern for each period under study. Those values are provided in the following table.

\begin{tabular}{ccccccccc}
\hline \hline Cohorts & 1820 & 1845 & 1870 & 1895 & 1920 & 1945 & 1970 & 1995 \\
$B_{t}$ & 700 & 460 & 540 & 700 & 780 & 550 & 2150 & 2650 \\
\hline \hline
\end{tabular}

Table A4: Calibration of TFP parameter $B_{t}$

Together, the calibrated values for all production parameters allow us to obtain a perfect fit of the GDP figures for France in the Maddison Project (2013) (see Figure A1) and a reasonable proxy for the headcount poverty rates for France in Ravallion (2016). Note that the reason why our fit is not perfect in the latter case is uniquely due to our reliance on a 10 income class model.

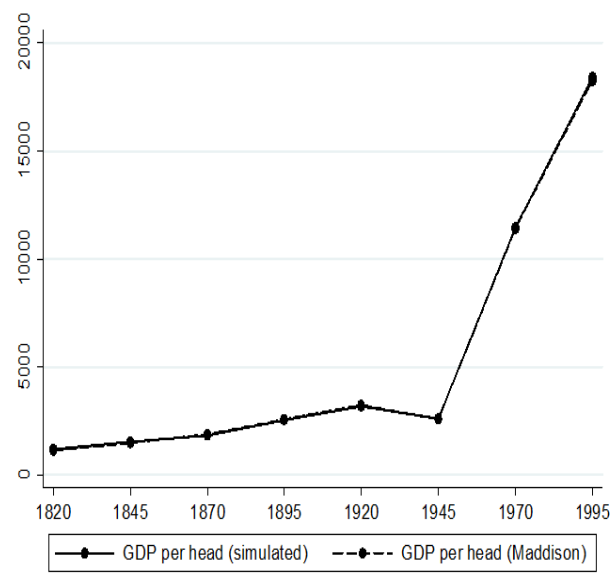

Figure A1: GDP per capita (Maddison 2013) and GDP per capita (simulated), France, 1820-1995.

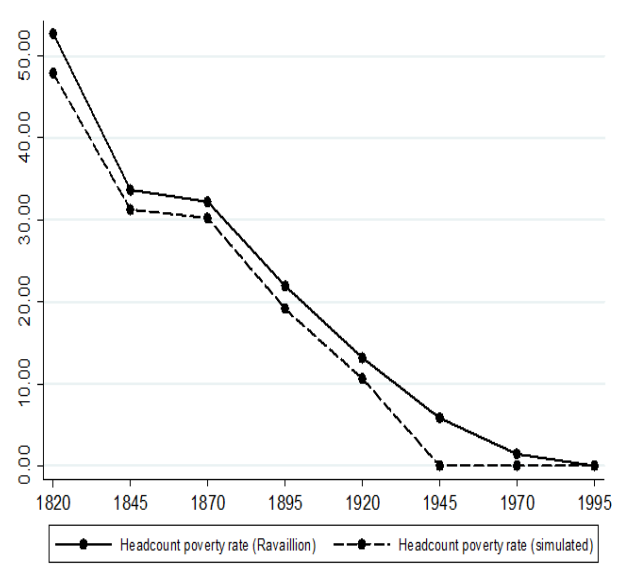

Figure A2: Headcount poverty rate (Ravallion 2016) and headcount poverty rate (simulated), France, 1820-1995. 


\subsection{Robustness}

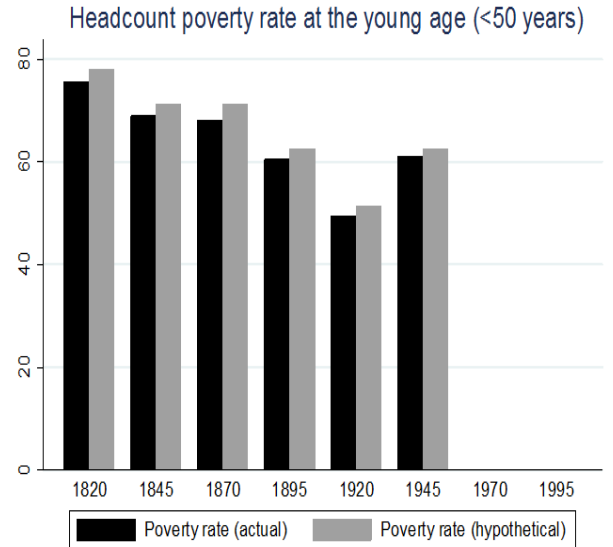

Figure A3: Comparison of actual and hypothetical absolute poverty rates for the young (poverty line $=\$ 6$ a day).

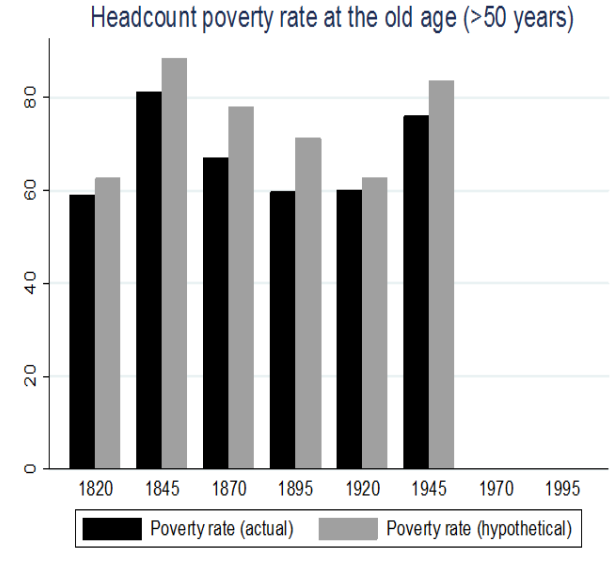

Figure A4: Comparison of actual and hypothetical absolute poverty rates for the old (poverty line $=\$ 6$ a day).
Relative headcount poverty rate at the young age ( $<50$ years)

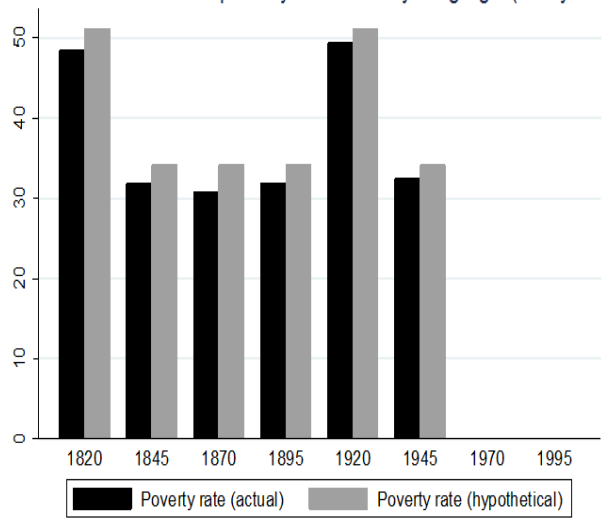

Figure A5: Comparison of actual and hypothetical relative poverty rates for the young (poverty line $=40 \%$ of median income).
Relative headcount poverty rate at the old age ( $>50$ years)

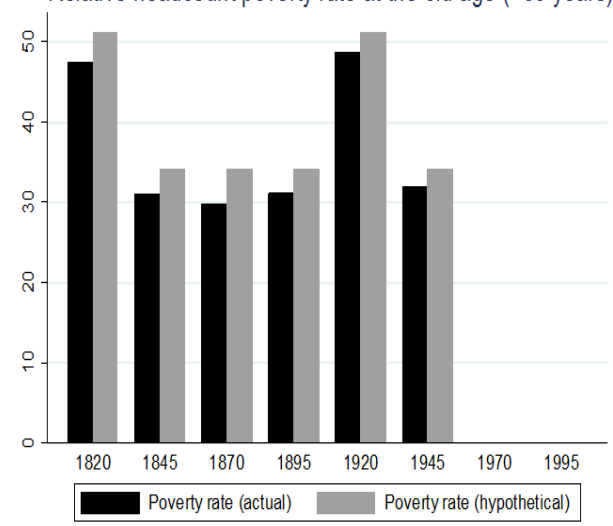

Figure A6: Comparison of actual and hypothetical relative poverty rates for the old (poverty line $=40 \%$ of median income). 